\title{
Some New Upper Bounds for the $Y$-Index of Graphs
}

\author{
Durbar Maji $\mathbb{D},{ }^{1}$ Ganesh Ghorai $\mathbb{D},{ }^{1}$ and Faria Ahmed Shami $\mathbb{D}^{2}$ \\ ${ }^{1}$ Department of Applied Mathematics with Oceanology and Computer Programming, Vidyasagar University, Midnapore 721102, \\ India \\ ${ }^{2}$ Department of Mathematics, Bangabandhu Sheikh Mujibur Rahman Science and Technology University, Gopalganj, Bangladesh
}

Correspondence should be addressed to Faria Ahmed Shami; fariashami@bsmrstu.edu.bd

Received 25 November 2021; Revised 1 January 2022; Accepted 3 January 2022; Published 30 January 2022

Academic Editor: Naeem Jan

Copyright (c) 2022 Durbar Maji et al. This is an open access article distributed under the Creative Commons Attribution License, which permits unrestricted use, distribution, and reproduction in any medium, provided the original work is properly cited.

\begin{abstract}
In mathematical chemistry, the topological indices with highly correlation factor play a leading role specifically for developing crucial information in QSPR/QSAR analysis. Recently, there exists a new graph invariant, namely, $Y$-index of graph proposed by Alameri as the sum of the fourth power of each and every vertex degree of that graph. The approximate range of the descriptors is determined by obtaining the bounds for the topological indices of graphs. In this paper, firstly, some upper bounds for the $Y$-index on trees with several types of domination number are studied. Secondly, some new bounds are also presented for this index of graphs in terms of relevant parameters with other topological indices. Additionally, a new idea on bounds for the $Y$-index by applying binary graph operations is computed.
\end{abstract}

\section{Introduction}

In this paper, we only consider the molecular graphs (MG) [1], which are simple and connected. In chemical graph theory, molecules or molecular compounds are often modelled by chemical structure as MG. The atoms of a molecular compound are to be represented as the vertices of the MG, whereas the edges represent the chemical bonds. Let $\mathscr{G}=$ $\mathscr{G}(V, E)$ be a MG with $V(\mathscr{G})=\left\{\mu_{1}, \mu_{2}, \ldots, \mu_{n}\right\}$ as the vertex set and $E(\mathscr{G})=\left\{e_{1}, e_{2}, \ldots, e_{m}\right\}$ as the edge set, such that $|V(\mathscr{G})|=n$ and $|E(\mathscr{G})|=m$. The degree of $\mu \in V(\mathscr{G})$, denoted by $\xi(\mu / \mathscr{G})$, is the total number of edges, which are associated with $\mu$. Obviously, $0 \leq \delta \leq \xi(\mu / \mathscr{G}) \leq \Delta \leq(n-1)$, where $\delta=\min \{\xi(\mu / \mathscr{G}) \mid \mu \in \mathscr{G}\}$ and $\Delta=\max \{\xi(\nu / \mathscr{G}) \mid \nu \in \mathscr{G}\}$. A set $S \subseteq V(\mathscr{G})$ satisfying the condition $\forall \mu \in V(\mathscr{G}) \backslash S, N_{\mathscr{G}}(\mu)$ $\cap S \neq \varnothing\}$ is called a dominating set of $\mathscr{G}$. When $\mathscr{R} \subseteq V(\mathscr{R})$ satisfies the condition $\forall \mu \in V(\mathscr{R}) \backslash \mathscr{R}, \xi_{\mathscr{R}}(\mu, \nu) \leq \kappa$ for some $\nu \in \mathscr{R}$, where $\xi_{\mathscr{R}}(\mu, \nu)$ denotes the distance between $\mu$ and $\nu$, is said to be a distance $\kappa$-domination $\left(D D_{k}\right)$ set of $\mathscr{R}$. The $\left(D D_{k}\right)$ number $([2,3])$ of $\mathscr{G}$, denoted by $\zeta_{k}$, is the minimum cardinality among all $\mathscr{R}$ sets. The notations $\operatorname{diam}(\mathscr{G})=\max$ $\left\{\varepsilon_{\mathscr{G}}(\mu) \mid \mu \in V(\mathscr{G})\right\} \quad$ and $\quad \varepsilon_{\mathscr{G}}(\mu)=\max \left\{\xi_{\mathscr{G}}(\mu, \nu) \mid \nu \in V(\mathscr{G})\right\}$ denote the diameter of $\mathscr{G}$ and the eccentricity of $\mu$, respectively. A path $P$ is called a diameter path $(D P)$ of $\mathscr{G}$ when the length of $P$ is equal to $\operatorname{diam}(\mathscr{G})$. We follow the book [4] for the graph theoretical definitions and notations.

The graph invariant (GI) is a number that is uniquely determined by a graph. The subset of (GI) $s$ is topological indices, which are used to predict several properties such as physical, chemical, pharmaceutical, and biological activities of chemical species. In 1947, the great chemist holder Wiener initiated a first-time idea about the topological index (TI). He presented the first TI, namely, Wiener index [5] to search the boiling points of alkanes. After long years, Gutman and Trinajstic [6] investigated two oldest GI $s$. They are defined as $M_{1}(\mathscr{G})=\sum_{\mu \in V(\mathscr{G})} \xi^{2}(\mu / \mathscr{G})$ and $M_{2}(\mathscr{G})=\sum_{\mu \nu \in E(\mathscr{G})} \xi(\mu / \mathscr{G}) \xi$ $(\nu / \mathscr{G})$, respectively. The concept of first general ZI was considered by Li and Zheng [7]. It is defined as

$$
M_{1}^{\lambda}(\mathscr{G})=\sum_{\mu \in V(\mathscr{G})} \xi^{\lambda}\left(\frac{\mu}{\mathscr{G}}\right)=\sum_{\mu \nu \in E(\mathscr{G})}\left[\xi^{\lambda-1}\left(\frac{\mu}{\mathscr{G}}\right)+\xi^{\lambda-1}\left(\frac{\nu}{\mathscr{G}}\right)\right]
$$

where $\lambda \in \mathfrak{R}-\{0,1\}$. For $\lambda=3$, it becomes forgotten topological index proposed by Furtula et al. [8]. It is presented as 


$$
F(\mathscr{G})=\sum_{\mu \in V(\mathscr{G})} \xi^{3}\left(\frac{\mu}{\mathscr{G}}\right)=\sum_{\mu \nu \in E(\mathscr{G})}\left[\xi^{2}\left(\frac{\mu}{\mathscr{G}}\right)+\xi^{2}\left(\frac{\nu}{G \mathscr{G}}\right)\right] .
$$

Liu et al. ([9]) introduced the reformulated $F$-index of $\mathscr{G}$ as follows:

$$
\mathrm{RF}(\mathscr{G})=\sum_{e \in E(\mathscr{G})} \xi^{3}\left(\frac{e}{\mathscr{G}}\right)=\sum_{e \sim f \in E(\mathscr{G})}\left[\xi^{2}\left(\frac{e}{\mathscr{G}}\right)+\xi^{2}\left(\frac{f}{\mathscr{G}}\right)\right] .
$$

In [10], Milicevic et al. introduced the first reformulated Zagreb index of a graph $\mathscr{G}$. It is defined as

$$
\operatorname{EM}_{1}(\mathscr{G})=\sum_{e \in E(\mathscr{G})} \xi^{2}(e)
$$

where $\xi(e)=\xi(\mu / \mathscr{G})+\xi(\nu / \mathscr{G})-2$. Recently, Alameri et al. [11] introduced a GI named $Y$-index (YI) and defined as

$$
Y(\mathscr{G})=\sum_{\mu \in V(\mathscr{G})} \xi^{4}\left(\frac{\mu}{\mathscr{G}}\right)=\sum_{\mu \nu \in E(\mathscr{G})}\left[\xi^{3}\left(\frac{\mu}{\mathscr{G}}\right)+\xi^{3}\left(\frac{\nu}{\mathscr{G}}\right)\right] .
$$

The YI is the special case of the first general Zagreb index for $\lambda=4$.

In this study, we obtain some new upper bounds (UB) for the YI in terms of different graph parameters, on $\zeta_{\kappa}(\mathscr{T})$ for tree of vertex $n$ and TI $s$. We arrange the remaining work as follows: Section 2 contains the UB for the YI on trees with $\zeta_{k}$. Section 3 contains some UB for YI in behavior of some relevant parameters. Section 4 collects some UB for YI under several graph operations. Finally, Section 5 presents the conclusions of the obtained results. To know more related to this field, readers are referred to [12-17].

\section{Preliminaries}

To establish the main results, the following lemmas are required.

Lemma 1. [18]). Let $\mathscr{T}$ be a $n(>3)$ vertex tree with $e=p_{1} p_{2} \in E(\mathscr{T})$, a nonpendant edge. Suppose the union of $\mathscr{T}_{1}$ and $\mathscr{T}_{2}$ is equal to $\mathscr{T}-p_{1} p_{2}$, where $p_{i} \in \mathscr{T}_{i}$ for $i \in\{1,2\}$. Let $\mathscr{T}$ be a new tree obtained by taking an edge joining transformation (EJT) of $\mathscr{T}$ on $e$. It is attained by identifying $p_{1} \in T_{1}$ with $p_{2} \in \mathscr{T}_{2}$ and also joining a pendent vertex $s$ to the $p\left(=p_{1}=p_{2}\right)$. In short, we denote $\mathscr{T}=\phi\left(\mathscr{T}, p_{1} p_{2}\right)$. Then, we get $Y(\mathscr{T})<Y(\mathscr{T})$.

Lemma 2. [19]). If $\mathscr{G}$ is an $n$ vertex $M G$ graph with $n=\kappa+1$, then $\zeta_{\kappa}(\mathscr{G})=\lfloor n / \kappa+1\rfloor$.

Lemma 3. [20]). Let $\mathscr{H}$ and $\mathscr{T}$ be two trees with $n$ and $(\kappa+$ 1) $n$ vertices, respectively. Then, $\zeta_{\kappa}(\mathscr{T})=n$ holds iff at least one of following conditions is satisfied:

(1) $\mathscr{T}$ is any $(\kappa+1)$-vertex tree.

(2) $\mathscr{T}$ is equal to $\mathscr{H}^{\circ} \kappa$ obtained by taking $\mathscr{H}$ and $n$ copies of $P_{\kappa-1}$ and then link with the $j^{\text {th }}$ vertex of $\mathscr{H}$ to exactly one end vertex in the $j^{\text {th }}$ copy of $P_{\kappa-1}$.
Lemma 4. [2]). If $\mathscr{G}$ contains the maximum value of the $Z I s$ among all $M G$ s of $n$-vertices with $\zeta_{\kappa}(\mathscr{G})$ and $S_{\mathscr{G}}=\{\mu \in V(\mathscr{G})$ $\left.\mid \xi(\mu / \mathscr{G})=1, \zeta_{\kappa}(\mathscr{G}-\mu)=\zeta_{\kappa}(\mathscr{G})\right\}$. If $S_{\mathscr{G}} \neq \varnothing$, then $\left|N_{\mathscr{G}}\left(S_{\mathscr{G}}\right)\right|$ $=1$.

Lemma 5. [2]). Suppose $\mu$ and $v$ be two vertices in $\mathscr{G}$ such that $p_{1}, p_{2}, \ldots, p_{r}$ and $q_{1}, q_{2}, \ldots, q_{t}$ pendent vertices adjacent to $p$ and $q$ respectively. Define $\mathscr{G}^{\prime}=\mathscr{G}-\left\{q q_{1}, q q_{2}\right.$, $\left.\ldots, q_{t}\right\}+\left\{p q_{1}, p q_{2}, \ldots, p q_{t}\right\}$ and $\mathscr{G}^{\prime \prime}=\mathscr{G}-\left\{p p_{1}, p p_{2}, \ldots\right.$, $\left.p p_{r}\right\}+\left\{q p_{1}, q p_{2}, \ldots, q p_{r}\right\}$. Then either $M_{i}\left(\mathscr{G}^{\prime}\right)$ is greater than $M_{i}(\mathscr{G})$ or $M_{i}\left(\mathscr{G}^{\prime \prime}\right)$ is greater than $M_{i}(\mathscr{G}), i=1,2$.

Lemma 6. [2]). Let $\mathscr{T}$ be a tree of order $n$ with $\Delta$ and $\zeta_{\kappa} \geq 2$. Then $\kappa \zeta_{\kappa}(\mathscr{T}) \leq(n-\Delta(\mathscr{T}))$.

Lemma 7. [21]). Suppose $\mu \nu$ is any edge of $\mathscr{G}$ with $n$ vertices. Then, for any integer $t \geq 2$

$$
\begin{aligned}
\text { (i) } \mid & |\xi(\mu / \mathscr{G}), \xi(\nu / \mathscr{G})|_{S(\mathscr{G}, t)}=n^{t-2}(n-\xi(\mu / \mathscr{G})-\xi(\nu / \mathscr{G}) \\
+ & (\mu, \nu)) \\
\text { (ii) } & |\xi(\mu / \mathscr{G}), \xi(\nu / \mathscr{G})+1|_{S(\mathscr{G}, t)}=n^{t-2}(\xi(\mu / \mathscr{G})-\triangleright(\mu, \nu))- \\
& \left.\beta(n)_{t-2} \xi(\mu / \mathscr{G})\right) \\
\text { (iii) } \mid & |\xi(\mu / \mathscr{G})+1, d(\nu / \mathscr{G})|_{S(\mathscr{G}, t)}=n^{t-2}(\xi(\mu / \mathscr{G})-\triangleright(\mu, \nu)) \\
& \left.-\beta(n)_{t-2} \xi(\nu / \mathscr{G})\right) \\
\text { (iv) } & |\xi(\mu / \mathscr{G})+1, \xi(\nu / \mathscr{G})+1|_{S(\mathscr{G}, t)}=n^{t-2}((\triangleright(\mu, \nu)+1)+ \\
& \left.\beta(n)_{t-2}(\xi(\mu / \mathscr{G})+\xi(\nu / \mathscr{G})+1)\right) .
\end{aligned}
$$

Lemma 8. [22]). (Radon's inequality) Let $x=\left(x_{i}\right)_{i=1}^{n}$ and $y=\left(y_{i}\right)_{i=1}^{n}$ be two sequences of positive real numbers. For any $\alpha \geq 0$,

$$
\sum_{i=1}^{n} \frac{x_{i}^{\alpha+1}}{y_{i}^{\alpha}} \geq \frac{\left(\sum_{i=1}^{n} x_{i}\right)^{\alpha+1}}{\left(\sum_{i=1}^{n} y_{i}\right)^{\alpha}}
$$

where the equality occurs for $x_{i}=p y_{i}$ for some constant $p$, for all $i=1,2, \ldots, n$.

\section{Main Results and Discussions}

3.1. Some UB for the YI on Trees with $D D_{\kappa}$ Number. In this section, we establish some sharp UB for the YI of graphs on the trees as to the $\mathrm{DD}_{\kappa}$ number, $\zeta_{\kappa}$. The set of all $n$ vertex trees with $\zeta_{\kappa}$ and the star of order $(n-\kappa \zeta+1)$ with $u_{1}, u_{2}$, $\ldots, u_{n-\kappa \zeta_{\kappa}}$ pendent vertices are denoted as $\mathscr{T}_{n, \kappa, \zeta_{\kappa}}$ and $S_{n-\kappa \zeta_{\kappa}+1}$, respectively.

Theorem 1. Let $\mathscr{T}$ be a tree of order $n$ and it contains $\zeta_{\kappa}(\mathscr{T})$ $=2$; then the $U B$ of $Y(\mathscr{T})$ can be expressed as $Y(\mathscr{T}) \leq(n$ $-2 \kappa)^{4}+(n-2(\kappa+1))+(16 \kappa+1)+16 \kappa-15$. The equality holds for $\mathscr{T} \Leftrightarrow \mathscr{T}_{n, \kappa, 2}^{j}$, where $j \in\{1,2, \ldots, k\}$.

Proof. Let $\mathscr{T} \in \mathscr{T}_{n, \kappa, 2}$ be a tree with a DP such that $P: u_{0}$, $u_{1}, \ldots, u_{d}$. For $d \leq 2 \kappa$, there exists a $\mathrm{DD}_{\kappa}$ set $\left\{\mu_{\lfloor d / 2\rfloor}\right\}$ of $\mathscr{T}$, a contradiction. In case $d \geq 2 \kappa+2$, also denoted by $\mathscr{T}=\phi\left(\mathscr{T}, u_{i} u_{i+1}\right)$ the tree obtained from $\mathscr{T}$ by EJT (Lemma $1)$ on the edge $u_{i} u_{i+1}$ for some $i \in\{1,2, \ldots, d-2\}$, then $\zeta_{\kappa}(\mathscr{T})=2$; therefore, $\mathscr{T} \in \mathscr{T}_{n, \kappa, 2}$. But $Y(\mathscr{T})>Y(\mathscr{T})$, a contradiction. Thus, it is only s for $d=2 \kappa+1$. 
In this case, we consider a tree $\mathscr{T}_{n, \kappa, 2}^{\alpha} \in \mathscr{T}_{n, \kappa, 2}$ obtained from the path $P_{2 \kappa+2}=w_{0} w_{1} \ldots w_{2 \kappa+1}$ by attaching $n-2(\kappa+$ 1) pendent vertices to $w_{\alpha}$, where $\alpha \in\{1, \ldots, 2 \kappa\}$. Moreover, $\mathscr{T}_{n, \kappa, 2}^{\alpha} \cong \mathscr{T}_{n, \kappa, 2}^{d-\alpha}$ for $\kappa+1 \leq \alpha \leq d-1$ and also $Y\left(\mathscr{T}_{n, \kappa, 2}^{\alpha}\right)=$ $Y\left(\mathscr{T}_{n, \kappa, 2}^{n, k, 2}\right)$ for $1 \leq \alpha \neq \beta \leq d-1$. Consequently, $\mathscr{T} \cong \mathscr{T}_{n, \kappa, 2}^{\alpha, 2}$, for some $\alpha \in\{1,2, \ldots, \kappa\}$. Therefore, the YI for the tree $\mathscr{T}$ can be directly computed as $Y(\mathscr{T})=Y\left(\mathscr{T}_{n, \kappa, 2}^{\alpha}\right)=(n-2 \kappa)^{4}+(n-$ $2(\kappa+1))+(16 \kappa+1)+16 \kappa-15$.

Theorem 2. Consider an $n$ vertex tree $\mathscr{T}$ that belongs to $\mathscr{T}_{n, \kappa, 3}$. Then, $Y(\mathscr{T}) \leq(n-3 \kappa)^{4}+(n-3(\kappa+1))+2(16 \kappa+1)$ $+16 \kappa-15$. The equality occurs as $\mathscr{T} \Leftrightarrow \mathscr{T}_{n, \kappa, 3}$.

Proof. Given that $\mathscr{T} \in \mathscr{T}_{n, \kappa, 3}$. Obviously $n \geq(\kappa+1) \zeta_{\kappa}$, by Lemma 2. Also, the equality $n=(\kappa+1) \zeta_{\kappa}$ of Lemma 3 holds the results. Now, we proceed to prove the theorem by induction hypothesis $(\mathrm{IH})$ on $n$. Assume that $n>3(\kappa+1)$ and the statement is true for $n-1$. Our main goal is to reach $\mathscr{T} \cong \mathscr{T}_{n, \kappa, 3}$.

Let $P=u_{0} u_{1} \ldots u_{d}$ and $\mathscr{D}$ be a DP and minimum $\mathrm{DD}_{\kappa}$ set of $\mathscr{T}$, respectively. Actually, we have to prove $d \geq 2 \kappa+2$. Otherwise, $\left\{u_{\kappa}, u_{\kappa+1}\right\}$ is a $\mathrm{DD}_{\kappa}$ set, a contradiction. Let us assume that $\left\{u_{\kappa}, u_{d-\kappa}\right\} \subseteq \mathscr{D}$ such that $\left(\cup_{r=0}^{\kappa} V\left(\mathscr{T}_{r}\right) \backslash\left\{u_{\kappa}\right\}\right)$ $\cap \mathscr{D}=\varnothing$ and $\left(\cup_{r=d-\kappa}^{d} V\left(\mathscr{T}_{r}\right) \backslash\left\{u_{d-\kappa}\right\}\right) \cap \mathscr{D}=\varnothing$.
Choose $u_{0}\left(=\alpha_{1}\right), u_{2}, \ldots, u_{d}\left(=\alpha_{t}\right)$ as the pendent vertices of $\mathscr{T}$ and also $\zeta_{\mathscr{T}}=\left\{\alpha_{i} \mid \zeta_{\kappa}\left(\mathscr{T}-\alpha_{i}\right)=\zeta_{\kappa}(\mathscr{T})\right\}$ for $1 \leq i \leq t$. If $\zeta_{\mathscr{T}}=\varnothing$, then $\zeta_{\mathscr{T}}=\left\{\alpha_{i} \mid \zeta_{\kappa}\left(\mathscr{T}-\alpha_{i}\right)=\zeta_{\kappa}(\mathscr{T})-1\right\}$ for $1 \leq i \leq t$.

When $\xi\left(u_{i} / \mathscr{T}\right) \geq 3$, then $\left\{\alpha_{2}, \ldots, \alpha_{t-1}\right\} \cap V\left(\mathscr{T}_{i}\right) \neq \varnothing$. Taking $\left\{u_{\kappa}, u_{d-\kappa}\right\} \in \mathscr{D}$, so $\zeta_{\kappa}(\mathscr{T}-x)=\zeta_{\kappa}(\mathscr{T})$ for $x \in\left\{\alpha_{2}\right.$, $\left.\ldots, \alpha_{t-1}\right\} \cap V\left(\mathscr{T}_{i}\right)$, a contradiction. Therefore $\xi\left(u_{i} / \mathscr{T}\right)=2$ for $i \in\{1,2, \ldots, \kappa, d-\kappa, \ldots, d-1\}$. Clearly $\zeta_{\kappa}\left(\mathscr{T}-u_{0}\right)=\zeta_{\kappa}$ $(\mathscr{T})-1$, since $\xi\left(u_{1} / \mathscr{T}\right)=2$.

Remark that $\xi_{\mathscr{T}}\left(u_{1}, u_{\kappa+1}\right)=\kappa$ and $\left(\cup_{r=0}^{\kappa} V\left(\mathscr{T}_{r}\right) v_{\kappa}\right) \cap \mathscr{D}$ $=\varnothing$. So, $u_{\kappa+1} \in \mathscr{D}$. Likewise, $u_{d-\kappa-1} \in \mathscr{D}$. For $d>2 \kappa+2$, the vertices $u_{\kappa}, u_{\kappa+1}, u_{d-\kappa-1}, u_{d-\kappa}$ are distinguished, a contradiction. So, $d=2 \kappa+2$ and $\mathscr{D}=\left\{u_{\kappa}, u_{\kappa+1}, u_{d-\kappa}\right\}$.

On the other side, if $\xi\left(u_{\kappa+1} / \mathscr{T}\right)=2$, then $\mathscr{T} \cong P_{2 \kappa+3}$ and $\left\{u_{\kappa}, u_{d-\kappa}\right\}$ is a $\mathrm{DD}_{\kappa}$ set, which is an inconsistency. Therefore, $\xi\left(u_{\kappa+1} / \mathscr{T}\right) \geq 3$ and also $\zeta_{\kappa}=3 \leq m$. When $m>3$, then $\zeta_{\kappa}\left(\mathscr{T}-\alpha_{i}\right)=\zeta_{\kappa}(\mathscr{T})$ for some $i \in\{1, \ldots, m\}$, an impropriety. So, $m=3$. Thus, $\mathscr{T}_{\kappa+1}$ is a path of which ended vertices are $u_{\kappa+1}$ and $\alpha_{3}$. That is, $\xi\left(u_{\kappa+1}, \alpha_{3}\right)=\kappa$. Hence, $|V(\mathscr{T})|=$ $3(\kappa+1)$, which contradicts $n>3(\kappa+1)$.

Assume that $v$ is a unique vertex $\alpha_{i}$ which is a pendent vertex with $\zeta_{\kappa}\left(\mathscr{T}-\alpha_{i}\right)=\zeta_{\kappa}(\mathscr{T})$. Note that $\xi(v / \mathscr{T}) \leq \Delta \leq$ $(n-3 \kappa)$, by Lemma 6 . So, by the IH and the definition of $Y(\mathscr{T})$, we get

$$
\begin{aligned}
Y(\mathscr{T}) & =Y\left(\mathscr{T}-\alpha_{i}\right)+4 \xi^{3}\left(\frac{v}{\mathscr{T}}\right)-6 \xi^{2}\left(\frac{v}{\mathscr{T}}\right)+4 \xi\left(\frac{v}{\mathscr{T}}\right) \\
& \leq(n-1-3 \kappa)^{4}+(n-1-3(\kappa+1))+2(16 \kappa+1)+16 \kappa-15+4(n-3 \kappa)^{3}-6(n-3 \kappa)^{2}+4(n-3 \kappa) \\
& =(n-3 \kappa)^{4}+(n-3(\kappa+1))+2(16 \kappa+1)+16 \kappa-15
\end{aligned}
$$

Therefore, the equality arrives if and only if $\mathscr{T}-\alpha_{i} \cong$ $\mathscr{T}_{n-1, \kappa, 3}$ and $\xi(v / T)=\Delta=(n-3 \kappa)$, that is, $\mathscr{T} \Leftrightarrow \mathscr{T}_{n, \kappa, 3}$.

Theorem 3. Let $\mathscr{T}$ be a tree having $n$ vertices and $\zeta_{k}(\mathscr{T}) \geq 3$. If $n=(\kappa+1) \zeta_{k}$, we have $Y(\mathscr{T}) \leq\left(\zeta_{\kappa}-1\right)^{4}+4\left(\zeta_{\kappa}-1\right)^{3}+$ $\left(\zeta_{\kappa}-1\right)\left(6 \zeta_{\kappa}+5\right)+2(8 \kappa-3) \zeta_{\kappa}-8$. The equality is attained when $\mathscr{T} \Leftrightarrow \mathscr{T}_{n, \kappa, \zeta_{\kappa}}$.
Proof. Given that $n=(\kappa+1) \zeta_{\kappa}$ for the tree $\mathscr{T}$ of $n$ vertices with $\mathrm{DD}_{\kappa}$ number, $\zeta_{\kappa}(\geq 3)$. By Lemma 3 , we get $\mathscr{T}=\mathscr{G}^{\circ} \kappa$ for some tree $\mathscr{G}$ on $\zeta_{\kappa}$ vertices. Let us consider $V(\mathscr{G})=$ $\left\{u_{1}, u_{2}, \ldots, u_{\kappa}\right\}$. Then, $\xi\left(u_{i} / \mathscr{G}\right)=\xi\left(v_{i} / T\right)-1$. Therefore, $\sum_{i=1}^{\zeta_{\kappa}} \xi\left(u_{i} / \mathscr{G}\right)=2\left(\zeta_{\kappa}-1\right)$ since for every tree (assume $\mathscr{T}^{\prime}$ ) containing $n$-vertices with vertex set $\left\{x_{1}, x_{2}, \ldots, x_{n}\right\}$ occurs $\sum_{i=1}^{\zeta_{\kappa}} \xi\left(x_{i} / \mathscr{T}^{\prime}\right)=2(n-1)$. By the definition of the YI, we can express

$$
\begin{aligned}
Y(\mathscr{T}) & =\sum_{i=1}^{\zeta_{\kappa}} \xi^{4}\left(\frac{u_{i}}{\mathscr{G}}\right)+\sum_{v_{i} \in V(\mathscr{T}) \backslash V(\mathscr{G})} \xi^{4}\left(\frac{v_{i}}{\mathscr{T}}\right) \\
& =\sum_{i=1}^{\zeta_{\kappa}}\left(\xi\left(\frac{u_{i}}{\mathscr{T}}\right)-1\right)^{4}+4 \sum_{i=1}^{\zeta_{\kappa}}\left(\xi\left(\frac{u_{i}}{\mathscr{T}}\right)-1\right)^{3}+6 \sum_{i=1}^{\zeta_{\kappa}}\left(\xi\left(\frac{u_{i}}{\mathscr{T}}\right)-1\right)^{2}+4 \sum_{i=1}^{\zeta_{\kappa}}\left(\xi\left(\frac{u_{i}}{\mathscr{T}}\right)-1\right)+\zeta_{\kappa}+16(\kappa-1) \zeta_{\kappa}+\zeta_{\kappa} \\
& =Y(\mathscr{G})+4 F(\mathscr{G})+6 M_{1}(\mathscr{G})+8\left(\zeta_{\kappa}-1\right)+2 \zeta_{\kappa}+16(\kappa-1) \zeta_{\kappa} \\
& \leq Y\left(S_{\zeta_{\kappa}}\right)+4 F\left(S_{\zeta_{\kappa}}\right)+6 M_{1}\left(\zeta_{\kappa}\right)+16 \kappa \zeta_{\kappa}-6 \zeta_{\kappa}-8 \\
& =\left(\zeta_{\kappa}-1\right)^{4}+4\left(\zeta_{\kappa}-1\right)^{3}+\left(\zeta_{\kappa}-1\right)\left(6 \zeta_{\kappa}+5\right)+2(8 \kappa-3) \zeta_{\kappa}-8
\end{aligned}
$$


for equalities $\mathscr{G} \Leftrightarrow S_{\zeta_{\kappa}}$ that imply $\mathscr{T} \Leftrightarrow \mathscr{T}_{n, \kappa, \zeta_{\kappa}}$.

Theorem 4. Consider $\mathscr{T}$ as an n-vertex tree whose $D D_{\kappa}$ number is $\zeta_{\kappa} \geq 3$. Then, $Y(\mathscr{T}) \leq\left(n-\kappa \zeta_{\kappa}\right)^{4}+\left(n-(\kappa+1) \zeta_{\kappa}\right)+$ $(16 \kappa+1)\left(\zeta_{\kappa}-1\right)+16 \kappa-15$.

The equality occurs for $\mathscr{T} \Leftrightarrow \mathscr{T}_{n, \kappa, \zeta}$.

Proof. Let $\mathscr{T} \Leftrightarrow \mathscr{T}_{n, \kappa, \zeta_{\kappa}}$ be a tree containing a DP such that $P=u_{0} u_{1} \ldots u_{d}$ that maximized the YI of graphs. The main goal is to establish the maximization of $Y(\mathscr{T})$ with respect to $\mathscr{T} \cong \mathscr{T}_{n, \kappa, \zeta_{\kappa}}$. Let us consider $\mathscr{D}$ to be a minimum $\mathrm{DD}_{\kappa}$ set of $\mathscr{T}$ and also define $\zeta_{\mathscr{T}}=\left\{x \in V(\mathscr{T}) \mid \xi(x / \mathscr{T})=1\right.$ and $\zeta_{\kappa}(\mathscr{T}$ $\left.-x)=\zeta_{\kappa}(\mathscr{T})\right\}$. If $\Gamma_{\mathscr{T}}=\varnothing$, then $\zeta_{\kappa}\left(\mathscr{T}-u_{i}\right)=\zeta_{\kappa}(\mathscr{T})-1$ for $i=0, d$. Also, for $\Gamma_{\mathscr{T}} \neq \varnothing$, by Lemma $4,\left|N_{\mathscr{T}}\left(\Gamma_{\mathscr{T}}\right)\right|=1$. In case, $u_{0}, u_{d} \in \Gamma_{\mathscr{T}}$, as $d-1>1$, we get $\left\{u_{1}, u_{d-1}\right\} \subseteq\left|N_{\mathscr{T}}\left(\Gamma_{\mathscr{T}}\right)\right|$ that implies $\left|N_{\mathscr{T}}\left(\Gamma_{\mathscr{T}}\right)\right|>1$, a contradiction. Therefore, we consider that $\zeta_{\kappa}\left(\mathscr{T}-u_{0}\right)=\zeta_{\kappa}(\mathscr{T})-1$, and thus $\left\{u_{\kappa}, u_{\kappa+1}\right.$, $\left.u_{d-\kappa}\right\} \subseteq \mathscr{D}$, from Theorem 2 .

By Lemma 1, applying EJT of $\mathscr{T}$ on any nonpendent edge of $\mathscr{T}_{\alpha}$ repeatedly for $\alpha=1, \ldots, \kappa$, it is to be constructed a tree $\mathscr{T}$ from $\mathscr{T}$ such that $\mathscr{T}_{\alpha} \cong S_{\left|V\left(\mathscr{T}_{\alpha}\right)\right|}$, where $\mathscr{T}_{\alpha}$ is the component of $\mathscr{T}-\left\{u_{\alpha-1} u_{\alpha}, u_{\alpha} u_{\alpha+1}\right\}$ having $u_{\alpha}$, for $\alpha=1$, $\ldots, \kappa$. Then, we have $\mathscr{T} \in \mathscr{T}_{n, \kappa, \zeta_{\kappa}}$ and also $Y(\mathscr{T}) \leq Y(\mathscr{T})$, where the equality holds $\mathscr{T} \Leftrightarrow \mathscr{T}$.

Now let $\mathscr{T}^{*}=\mathscr{T}-\cup_{\alpha \in\{1, \ldots, \kappa\} \backslash\left(\alpha_{r}\right)}\left\{u_{\alpha} w \mid w \in N_{\mathscr{T}}\left(u_{\alpha}\right) \backslash\right.$ $\left.\left\{u_{\alpha-1}, \quad u_{\alpha+1}\right\}\right\}+U_{\alpha_{r} \in\{1, \ldots, \kappa\} \backslash\left(\alpha_{r}\right)}\left\{u_{\alpha} w \| w \in N_{\mathscr{T}}\left(u_{\alpha}\right) \backslash\left\{u_{\alpha-1}\right.\right.$, $\left.\left.u_{\alpha+1}\right\}\right\}$ for some $\alpha_{r} \in\{1, \ldots, \kappa\}$.

Then, by Lemma 5 , we get $Y(\mathscr{T}) \leq \mathscr{T}^{*}$ with equality if and only if $\mathscr{T} \cong Y\left(\mathscr{T}^{*}\right)$.

Again, define by $\mathscr{T}^{\star}=\mathscr{T}^{*}-\left\{u_{\alpha_{r}} w \mid w \in N_{\mathscr{T}^{*}}\left(u_{\alpha_{r}}\right) \backslash\right.$ $\left.\left\{u_{\alpha_{r}-1}, u_{\alpha_{r}+1}\right\}\right\}+\left\{u_{\kappa+1} w \mid w \in N_{\mathscr{T}^{*}}\left(u_{\alpha_{r}}\right) \backslash\left\{u_{\alpha_{r}-1}, u_{\alpha_{r}+1}\right\}\right\}$. In fact, let $\left|N_{\mathscr{T}^{*}}\left(u_{\alpha_{r}}\right) \backslash\left\{u_{\alpha_{r}-1}, u_{\alpha_{r}+1}\right\}\right|=p, p \geq 0$.

Then, $\xi\left(u_{\alpha} / \mathscr{T}^{\star}\right)=2$ for $\alpha=1, \ldots, \kappa$ and also $\mathscr{D}$ will be the minimum $\mathrm{DD}_{\kappa}$ set of $\mathscr{T}^{\star}$. It implies that all the vertices in $\cup_{\alpha=0}^{k} N_{\mathscr{T}^{\alpha}}\left(u_{\kappa}\right) \backslash\left\{u_{0}, \ldots, u_{\kappa}\right\}$ can be determined by $u_{\kappa+1} \in \mathscr{D}$. Therefore, $\mathscr{D} \backslash\left\{u_{\kappa}\right\}$ will be a $\mathrm{DD}_{\kappa}$ set of $\mathscr{T}^{\star}-u_{0}, \ldots, u_{\kappa}$. Suppose that $\mathrm{PN}_{\kappa, \mathscr{D}}(y)$ is the set of all private $\kappa$-neighbors of $y$ upon $\mathscr{D}$ in $\mathscr{T}^{\star}$. Then, $\mathrm{PN}_{\kappa, \mathscr{D}}\left(u_{\kappa+1}\right) \subseteq V\left(\mathscr{T}^{\star}\right) \backslash u_{0}, \ldots, u_{\kappa}$. Thus, $\mathscr{D} \backslash\left\{u_{\kappa}\right\}$ will be a minimum $\mathrm{DD}_{\kappa}$ set of the tree $\mathscr{T}^{\star}-\left\{u_{0}, \ldots, u_{\kappa}\right\}$. Therefore, $\zeta_{\kappa}\left(\mathscr{T}^{\star}-\left\{u_{0}, \ldots, u_{\kappa}\right\}\right)=\zeta_{\kappa}-$ $1=\zeta_{\kappa}\left(\mathscr{T}^{\star}-\left\{u_{0}, \ldots, u_{\kappa-1}\right\}\right)$.

So, from the definition of YI, we have

$$
\begin{aligned}
Y\left(\mathscr{T}^{\star}\right)-Y\left(\mathscr{T}^{\star}\right) & =\left(d\left(\frac{u_{\kappa+1}}{\mathscr{T}^{*}}\right)+p\right)^{4}+2^{4}-\xi^{4}\left(\frac{u_{\kappa+1}}{\mathscr{T}^{*}}\right)-(p+2)^{4} \\
& =2 p\left(2 \xi^{3}\left(\frac{u_{\kappa+1}}{\mathscr{T}^{*}}\right)+3 p \xi^{2}\left(\frac{u_{\kappa+1}}{\mathscr{T}^{*}}\right)+2 p^{2} \xi\left(\frac{u_{\kappa+1}}{\mathscr{T}^{*}}\right)-4 p^{2}-12 p-16\right) \geq 0
\end{aligned}
$$

It means that $Y\left(\mathscr{T}^{\star}\right) \geq Y\left(\mathscr{T}^{\star}\right)$, where the equality holds iff either $p=0$ i.e. $\mathscr{T}^{*} \cong \mathscr{T}^{\star}$ or $\xi\left(u_{\kappa+1} / \mathscr{T}^{*}\right)=2$.

So far, we have proved $Y\left(\mathscr{T}^{\star}\right) \leq\left(n-\kappa \zeta_{\kappa}\right)^{4}+(n-(\kappa+$ 1) $\left.\zeta_{\kappa}\right)+(16 \kappa+1)\left(\zeta_{\kappa}-1\right)+16 \kappa-15$ with equality iff $\mathscr{T}^{\star} \cong$ $\mathscr{T}_{n, \kappa, \zeta_{\kappa}}$ by induction on $\zeta_{k}$. We have from Theorem 2 that the assertion is mathematics for $n \geq(\kappa+1) \zeta_{\kappa}$ as well as $\zeta_{k}=3$.
Now let us consider the affirmation contains for $\zeta_{k}-1$ and all the vertices $n \geq(\kappa+1)\left(\zeta_{\kappa}-1\right)$.

Because of $\zeta_{k}\left(\mathscr{T}^{\star}-\left\{u_{0}, \ldots, u_{\kappa}\right\}\right)$ and $\mid V\left(\mathscr{T}^{\star}\right)-\left\{u_{0}, \ldots\right.$, $\left.u_{\kappa}\right\} \mid=(n-\kappa-1) \geq(\kappa+1)\left(\zeta_{\kappa}-1\right)$, we get by the IH

$$
\begin{aligned}
Y\left(\mathscr{T}^{\star}\right) & =Y\left(\mathscr{T}^{*}-\left\{u_{0}, \ldots, u_{\kappa}\right\}\right)+4 \xi^{3}\left(u_{\kappa+1}\right)-6 \xi^{2}\left(u_{\kappa+1}\right)+4 \xi\left(u_{\kappa+1}\right)-1+\sum_{\alpha=0}^{\kappa} \xi_{\mathscr{T}^{*}}^{4}\left(u_{\alpha}\right) \\
& =Y\left(\mathscr{T}_{n-\kappa-1, \kappa, \zeta_{\kappa}-1}\right)+4\left(n-\kappa \zeta_{\kappa}\right)^{3}-6\left(n-\kappa \zeta_{\kappa}\right)^{2}+4\left(n-\kappa \zeta_{\kappa}\right)+16 \kappa \\
& =\left(n-\kappa \zeta_{\kappa}\right)^{4}+\left(n-(\kappa+1) \zeta_{\kappa}\right)+(16 \kappa+1)\left(\zeta_{\kappa}-1\right)+16 \kappa-15,
\end{aligned}
$$

where the equality holds iff $\mathscr{T}^{\star}-\left\{u_{0}, \ldots, u_{\kappa}\right\} \cong \mathscr{T}_{n-\kappa-1, \kappa, \zeta_{\kappa}-1}$ and also $\xi_{\mathscr{T}^{\star}}\left(u_{\kappa+1}\right)=\Delta=n-\kappa \zeta_{\kappa}$ and otherwise $\mathscr{T}^{\star} \cong$ $\mathscr{T}_{n, \kappa, \zeta_{\kappa}}$ with $\xi_{\mathscr{T}^{*}}\left(u_{\alpha}\right)=2$ for $\alpha=1, \ldots, \kappa$. Therefore, we can conclude that $Y(\mathscr{T}) \leq Y(\mathscr{T}) \leq Y\left(\mathscr{T}^{*}\right) \leq Y\left(\mathscr{T}^{*}\right) \leq \quad(n-$ $\left.\kappa \zeta_{\kappa}\right)^{4}+\left(n-(\kappa+1) \zeta_{\kappa}\right)+(16 \kappa+1)\left(\zeta_{\kappa}-1\right)+16 \kappa-15$ with either equality iff $\mathscr{T} \cong \mathscr{T} \cong \mathscr{T}^{*} \cong \mathscr{T}^{\star} \cong \mathscr{T}_{n, \kappa, \zeta_{\kappa}}$ or $\mathscr{T} \cong \mathscr{T} \cong$ $\mathscr{T}^{*}$ with $\xi_{\mathscr{T}^{*}}\left(u_{\kappa+1}\right)=2$. Besides, $\mathscr{T}^{\star} \cong \mathscr{T}_{n, \kappa, \zeta_{\kappa}}$.

Here, we determine some UB on the YI of trees containing $n$-vertices with domination number $\zeta$ ([23]). The $\mathrm{DD}_{\kappa}$ number of a graph is said to be the domination number of that graph if $\kappa=1$. If $\mathscr{T}$ is an $n$-vertex tree containing a DP such that $P: u_{0} u_{1} \ldots u_{d}$, then denote by $\mathscr{T}_{i}$ the component of $\mathscr{T}-\left\{u_{i-1} u_{i}, u_{i} u_{i+1}\right\}$ containing $u_{i}, i=1,2, \ldots, d-1$. To compute our main outcome, at first, we will focus on the following definition.

Consider $\mathscr{T}_{n, \zeta}$ to be a tree constructed from a star $K_{1, n-1}$ with involvement of a pendant edge to its $\zeta-1$ pendant vertices. Note that $\mathscr{T}_{n, \zeta} \in \mathscr{T}_{n, \zeta}$, a class of $n$ vertex trees and domination number $\zeta$. Also, $\zeta=1$ occurs iff $\mathscr{T} \cong \mathscr{T}_{1, n-1}$.

Corollary 1. If $\mathscr{T} \in \mathscr{T}_{n, \zeta}$, then $Y(\mathscr{T}) \leq(n-\zeta)^{4}+(n-2 \zeta+$ $1)+17(\zeta-1)$ with equality holding for $\mathscr{T} \Leftrightarrow \mathscr{T}_{n, \zeta}$. 
Proof. For $\Delta=2$, it occurs that $\mathscr{T} \cong P_{n}(n \geq 2)$. The equality holds for $\mathscr{T} \cong \mathscr{T}_{2,1}\left(\equiv P_{2}\right), \mathscr{T} \cong \mathscr{T}_{3,1}\left(\equiv P_{3}\right)$ and $\mathscr{T} \cong \mathscr{T}_{4,2}$ $\left(\equiv P_{4}\right)$. But for $n \geq 5$, the above inequality is strict. Now, we consider a diameter path $P=u_{0}, u_{1}, \ldots, u_{d}$ and a minimum dominating set $\mathscr{D}$ of $\mathscr{T}$ with $\Delta \geq 3$. To prove the theorem, we will take the way of $I H$ on $n$. Let us consider that Theorem 1 is true for $n-1$ and also the statement is to be proved as well as truth by replacing $n+1$ from $n$. When $\zeta\left(\mathscr{T}-\left\{u_{0}\right\}\right)=$ $\zeta(\mathscr{T})$, then by the $\mathrm{IH}$ we have

$$
Y(\mathscr{T})=Y\left(\mathscr{T}-\left\{u_{0}\right\}\right)+4 \xi^{3}\left(\frac{u_{1}}{\mathscr{T}}\right)-6 \xi^{2}\left(\frac{u_{1}}{\mathscr{T}}\right)+4 \xi\left(\frac{u_{1}}{\mathscr{T}}\right)
$$

$\leq(n-1-\zeta)^{4}+(n-2 \zeta)+17(\zeta-1)+4(n-\zeta)^{3}-6(n-\zeta)^{2}$ $+4 \xi(n-\zeta)($ since $\xi(u / \mathscr{T}) \leq n-\zeta$, by Lemma 6$)=(n-$ $\zeta)^{4}+(n-2 \zeta+1)+17(\zeta-1)$. The equality holds iff the pendant vertex $u_{0}$ is adjacent to the vertex $u_{1}$ of degree $\Delta=n-\zeta$, that is, $\mathscr{T} \cong \mathscr{T}_{n, \zeta}$.

Otherwise, assume that $\zeta\left(\mathscr{T}-\left\{u_{0}\right\}\right)=\zeta(\mathscr{T})-1$. So, it will be $\xi\left(u_{1} / \mathscr{T}\right)=2$ which also implies that $u_{1}$ belongs to every minimum dominating set, i.e., $\zeta\left(\mathscr{T}-\left\{u_{0}\right\}\right)=\zeta(\mathscr{T})$. Therefore, we can obtain by the $\mathrm{IH}$

$$
\begin{aligned}
Y(\mathscr{T}) & =Y\left(\mathscr{T}-\left\{u_{0}\right\}\right)+4 \xi^{3}\left(u_{1} / \mathscr{T}\right)-6 \xi^{2}\left(u_{1} / \mathscr{T}\right)+4 \xi\left(u_{1} / \mathscr{T}\right) \\
& \leq(n-\zeta+1)^{4}+(n-2 \zeta+3)+17(\zeta-2)+16 \\
& =(n-\zeta)^{4}+(n-2 \zeta+1)+17(\zeta-1),
\end{aligned}
$$

where the equality holds iff $\mathscr{T}-\left\{u_{0}\right\} \cong \mathscr{T}_{n-1, \zeta-1}$ and pendant vertex $u_{0}$ is adjacent to the vertex $u_{1}$ of degree 2 , that is, $\mathscr{T} \cong \mathscr{T}_{n, \zeta}$.

3.2. Some UB for the YI of Graphs with respect to Some Standard Parameters and Others TI. In this section, we establish the some sharp UB for the YI of $\mathscr{G} \cdot w \cdot r$ to some graph parameters such as $n, m, \delta, \Delta$ and others TI such as $M_{1}(\mathscr{G}), M_{2}(\mathscr{G}), F(\mathscr{G}), \mathrm{EF}(\mathscr{G}), \mathrm{EM}_{1}(\mathscr{G})$. Let $\mathscr{D}(\mathscr{G})=\{\xi$ $\left.\left(u_{1} / \mathscr{G}\right), \xi\left(u_{1} / \mathscr{G}\right), \ldots, \xi\left(u_{n} / \mathscr{G}\right)\right\}$. If $\mathscr{D}(\mathscr{G})=\{r\}$, then $\mathscr{G}$ is said to be $r$-regular. If $\mathscr{D}(\mathscr{G})=\{r, s\}$, then $\mathscr{G}$ is $(r, s)$ biregular and so on. Motivating the proof technique as in [24], we obtain an UB for YI in the following theorem.

Theorem 5. Consider $\mathscr{G}$ to be a $(n, m)$ graph, i.e., $\mathscr{G}$ contains $n$ vertices and $m$ edges. Then $Y(\mathscr{G}) \leq 2 m(\delta+\Delta)\left(\delta^{2}+\Delta^{2}\right)-$ $n \delta \Delta\left(\delta^{2}+\delta \Delta+\Delta^{2}\right)+(\delta-t)\left(\Delta^{3}+\delta \Delta^{2}+\delta \Delta\right)-t\left(\delta^{2}+t \delta+\right.$ $\left.t^{2}\right)$, where $t$ is the integer defined by relation $2 m-n \delta \equiv t-$ $\delta(\bmod (\Delta-\delta)), \quad \delta \leq t \leq \Delta-1$ and the equality holds iff at most one vertex of $\mathscr{G}$ has different degree from $\delta$ and $\Delta$.

Proof. Consider $x_{i}$ as the number of vertices of degree $i$ in $\mathscr{G}$. From the definition of YI, we can write

$$
Y(\mathscr{G})=\sum_{i=\delta}^{\Delta} i^{4} x_{i}
$$

Obviously,

$$
\begin{gathered}
\sum_{i=\delta}^{\Delta} i x_{i}=2 m, \\
\sum_{i=\delta}^{\Delta} x_{i}=n .
\end{gathered}
$$

After calculation, we get

$$
\begin{aligned}
& x_{\delta}=\frac{1}{\Delta-\delta}\left[n \Delta-2 m+\sum_{i=\delta+1}^{\Delta+1}(i-\Delta) x_{i}\right], \\
& x_{\Delta}=\frac{1}{\Delta-\delta}\left[2 m-n \delta+\sum_{i=\delta+1}^{\Delta+1}(\delta-i) x_{i}\right] .
\end{aligned}
$$

Using (15) and (16), we have

$$
\begin{aligned}
Y(\mathscr{G}) & =\delta^{4} x_{\delta}+\Delta^{4} x_{\Delta}+\sum_{i=\delta+1}^{\Delta-1} i^{4} x_{i} \\
& =\frac{\delta^{4}}{\Delta-\delta}\left[n \Delta-2 m+\sum_{i=\delta+1}^{\Delta+1}(i-\Delta) x_{i}\right]+\frac{\Delta^{4}}{\Delta-\delta}\left[2 m-n \delta+\sum_{i=\delta+1}^{\Delta+1}(\delta-i) x_{i}\right]+\sum_{i=\delta+1}^{\Delta-1} i^{4} x_{i} . \\
& =\frac{1}{\Delta-\delta}\left[\delta^{4}(n \Delta-2 m)+\Delta^{4}(2 m-n \delta)\right]+\frac{1}{\Delta-\delta} \sum_{i=\delta+1}^{\Delta-1}\left[\delta^{4}(i-\Delta)+\Delta^{4}(\delta-i)+i^{4}(\Delta-\delta)\right] x_{i}
\end{aligned}
$$


Actually, the term $\delta^{4}(i-\Delta)+\Delta^{4}(\delta-i)+i^{4}(\Delta-\delta)$ will be negative for $\delta+1 \leq i \leq \Delta-1$. So, the $Y(\mathscr{G})$ will be maximum if $x_{i}=0$ for $i=\delta+1, \ldots, \Delta-1$. Therefore, (15) and (16) become to $x_{\delta}=n \Delta-2 m / \Delta-\delta$ and $x_{\Delta}=2 m-$ $n \delta / \Delta-\delta$. These two equations require that

$$
2 m-n \delta \equiv 0(\bmod (\Delta-\delta)) .
$$

If the requirement is not true, we choose $x_{i}$ such that $n_{t}=1$ and $x_{i}=0$ for all $i=\delta+1, \ldots, \Delta-1$, except for $i=t$. Then, (15) and (16) become $x_{\delta}=n \Delta-2 m+k-\Delta / \Delta-\delta$ and $x_{\Delta}=2 m-(n-1) \delta / \Delta-\delta$ which satisfy conditions 3 and 4 . In [25], there survives a $n$ vertex graph $\mathscr{G}$ of which one vertex with degree 0 when $n$ and $\delta$ are both odd. If we add the edges to this graph, the vertex degrees increase one at a time up to $\Delta$. There occurs $2 m-n \delta \equiv t-\delta(\bmod (\Delta-\delta))$ that implies that the degree of one more vertex may be increased up to $t$. Therefore, there exists a graph of order $n$ and size $m$ along with a unique vertex of degree $t$ that different from $\delta$ and $\Delta$.

Suppose now that the graph $\mathscr{G}$ contains two vertices of degrees $i$ and $k$ for $\delta+1 \leq i \leq k \leq \Delta-1$. If the sum of vertex degrees remains the same by reducing the first vertex degree by 1 and increasing the second vertex degree by 1 , the value of the YI is replaced by

$$
\begin{aligned}
((\delta- & \left.(i-1))\left(\Delta^{3}+\delta \Delta^{2}+\delta^{2} \Delta-(i-1)\left(\delta^{2}+(i-1) \delta+(i-1)^{2}\right)\right)\right)-(\delta-i)\left(\Delta^{3}+\delta \Delta^{2}+\delta^{2} \Delta-i\left(\delta^{2}+i \delta+i^{2}\right)\right) \\
& +(\delta-(k+1))\left(\Delta^{3}+\delta \Delta^{2}+\delta^{2} \Delta-(k+1)\left(\delta^{2}+(k+1) \delta+(k+1)^{2}\right)\right)-(\delta-k)\left(\Delta^{3}+\delta \Delta^{2}+\delta^{2} \Delta-k\left(\delta^{2}+k \delta+k^{2}\right)\right) \\
& =(k+1)^{3}-(i-1)^{3}+3\left(k^{3}-i^{3}\right)+3\left(k^{2}+i^{2}\right)+(k-i)>0 .
\end{aligned}
$$

It means that condition 8 is not true, and it will be the optimal choice of the quantities $x_{i}=0$ for $\delta+1 \leq i \leq \Delta-1$ such that $x_{t}=1$ (except for $\left.i=t\right)$. Therefore, we can conclude from (17) that

$$
Y(\mathscr{G}) \leq 2 m(\delta+\Delta)\left(\delta^{2}+\Delta^{2}\right)-n \delta \Delta\left(\delta^{2}+\delta \Delta+\Delta^{2}\right)+(\delta-t)\left(\Delta^{3}+\delta \Delta^{2}+\delta \Delta\right)-t\left(\delta^{2}+t \delta+t^{2}\right) .
$$

Theorem 6. If $\mathscr{G}$ is a $(n, m)$ graph, then

$Y(\mathscr{G}) \leq E F(\mathscr{G})+6 E M_{1}(\mathscr{G})+12 M_{1}(\mathscr{G})-\frac{3 M_{1}^{2}(\mathscr{G})}{n}-16 m$, where the equality holds iff either $\mathscr{G}$ is regular or semiregular bipartite graph.

Proof. Using Lemma 8, for $\alpha=1$, setting $x_{i}=\xi(j / \mathscr{G})$ $+\xi(k / \mathscr{G})$ and $y_{i}=1 / \xi(j / \mathscr{G})+1 / \xi(k / \mathscr{G})$ for the graph $\mathscr{G}$ in (1), we have

$$
\begin{aligned}
& \sum_{j k \in E(\mathscr{G})} \frac{(\xi(j / \mathscr{G})+\xi(k / \mathscr{G}))^{2}}{1 / \xi(j / \mathscr{G})+1 / \xi(k / \mathscr{G})} \geq \frac{\left(\sum_{j k \in E(\mathscr{G})} \xi(j / \mathscr{G})+\xi(k / \mathscr{G})\right)^{2}}{\sum_{j k \in E(\mathscr{G})}(1 / \xi(j / \mathscr{G})+1 / \xi(k / \mathscr{G}))} \\
& \text { i.e. } \sum_{j k \in E(\mathscr{G})} \xi\left(\frac{j}{G}\right) \xi\left(\frac{k}{G}\right)\left(\xi\left(\frac{j}{G}\right)+\left(\frac{k}{G}\right)\right) \geq \frac{M_{1}^{2}(\mathscr{G})}{n} .
\end{aligned}
$$

Also,

$$
\begin{aligned}
& \sum_{j k \in E(\mathscr{G})} \xi\left(\frac{j}{\mathscr{G}}\right) \xi\left(\frac{k}{\mathscr{G}}\right)\left(\xi\left(\frac{j}{\mathscr{G}}\right)+\xi\left(\frac{k}{\mathscr{G}}\right)\right. \\
& =\frac{1}{3}\left[\sum_{j k \in E(\mathscr{G})}\left(\xi\left(\frac{j}{\mathscr{G}}\right)+\xi\left(\frac{k}{\mathscr{G}}\right)\right)^{3}-\sum_{j k \in E(\mathscr{G})}\left(\xi^{3}\left(\frac{j}{\mathscr{G}}\right)+\xi^{3}\left(\frac{k}{\mathscr{G}}\right)\right)\right]
\end{aligned}
$$




$$
\begin{aligned}
& =\frac{1}{3}\left[\sum_{e \in E(\mathscr{G})}\left(\xi\left(\frac{e}{\mathscr{G}}\right)+2\right)^{3}-Y(\mathscr{G})\right] ; \quad \text { where } \xi\left(e=\frac{i j}{\mathscr{G}}\right)=\xi\left(\frac{i}{\mathscr{G}}\right)+\xi\left(\frac{j}{\mathscr{G}}\right)-2 . \\
& =\frac{1}{3} \sum_{e \in E(\mathscr{G})} \xi^{3}\left(\frac{e}{\mathscr{G}}\right)+2 \sum_{e \in E(\mathscr{G})} \xi^{2}\left(\frac{e}{\mathscr{G}}\right)+4 \sum_{e \in E(\mathscr{G})} \xi\left(\frac{e}{\mathscr{G}}\right)+\frac{8}{3} m-\frac{1}{3} \sum_{j k \in E(\mathscr{G})}\left[\xi^{3}\left(\frac{j}{\mathscr{G}}\right)+\xi^{3}\left(\frac{k}{\mathscr{G}}\right)\right] \\
& =\frac{1}{3} E F(\mathscr{G})+2 E M_{1}(\mathscr{G})+4\left(M_{1}(\mathscr{G})-2 m\right)+\frac{8}{3} m-\frac{1}{3} Y(\mathscr{G}) .
\end{aligned}
$$

since $\sum_{e \in E(\mathscr{G})} \xi(e / \mathscr{G})=\sum_{i \in V(L(\mathscr{G}))} \xi(i / L(\mathscr{G}))=M_{1}(\mathscr{G})-2 m$.

From $(23)$ and $(24), M_{1}^{2}(\mathscr{G}) / n \leq 1 / 3 E F(\mathscr{G})+2 E M_{1}$ $(\mathscr{G})+4\left(M_{1}(\mathscr{G})-2 m\right)+8 / 3 m-1 / 3 Y(\mathscr{G})$.

Theorem 7. Suppose a graph $\mathscr{G}$ that contains $n$ vertices and $m$ edges. Then, $Y(\mathscr{G}) \leq M_{1}(\mathscr{G})\left(F(\mathscr{G})-M_{2}(\mathscr{G})\right)$, with equalities $\mathscr{G} \Leftrightarrow P_{2}$.
Proof. If $(a),(b), \ldots,(l)$ are positive numbers sets with $m$ elements in each set and $p, q, \ldots, t$ are positive numbers such that $p+q+\cdots+t>1$, then by Jensen's theorem $\sum_{i=1}^{m}\left(a_{i}^{p} b_{i}^{q} \ldots l_{i}^{t}\right) \leq\left(\sum_{i=1}^{m} a_{i}\right)^{p}\left(\sum_{i=1}^{m} b_{i}\right)^{q} \ldots\left(\sum_{i=1}^{m} l_{i}\right)^{t} . \quad$ We know

$$
\begin{aligned}
Y(\mathscr{G}) & =\sum_{u v \in E(\mathscr{G})}\left[\xi^{3}\left(\frac{u}{\mathscr{G}}\right)+\xi^{3}\left(\frac{v}{\mathscr{G}}\right)\right]=\sum_{u v \in E(\mathscr{G})}\left[\xi\left(\frac{u}{\mathscr{G}}\right)+\xi\left(\frac{v}{\mathscr{G}}\right)\right]\left[\xi^{2}\left(\frac{u}{\mathscr{G}}\right)+\xi^{2}\left(\frac{v}{\mathscr{G}}\right)-\xi\left(\frac{u}{\mathscr{G}}\right) \xi\left(\frac{v}{\mathscr{G}}\right)\right] \\
& \leq \sum_{u v \in E(\mathscr{G})}\left[\xi\left(\frac{u}{\mathscr{G}}\right)+\xi\left(\frac{v}{\mathscr{G}}\right)\right] \sum_{u v \in E(\mathscr{G})}\left[\xi^{2}\left(\frac{u}{\mathscr{G}}\right)+\xi^{2}\left(\frac{v}{\mathscr{G}}\right)-\xi\left(\frac{u}{\mathscr{G}}\right) \xi\left(\frac{v}{\mathscr{G}}\right)\right] .
\end{aligned}
$$

Setting $a_{i}=\xi(u / \mathscr{G})+\xi(v / \mathscr{G})$ and $b_{i}=\xi^{2}(u / \mathscr{G})+\xi^{2}(v /$ $\mathscr{G})-\xi(u / \mathscr{G}) \xi(v / \mathscr{G})$ and $p=q=1$, then by Jensen's theorem $=M_{1}(\mathscr{G})\left(F(\mathscr{G})-M_{2}(\mathscr{G})\right)$.

Theorem 8. Let $\mathscr{G}$ be a graph of $n$ order and $m$ size. Then, $Y(\mathscr{G}) \leq 16 m^{4} / \sqrt[3]{n^{16}}\left\{1 / 4(\Delta / \delta)^{16 / 3}+4 / 3 \delta / \Delta\right\}^{4}$. The equality occurred when $\mathscr{G}$ is regular graph.

Proof. We prove the theorem using the following inequalities. then

$$
\left(\sum_{i=1}^{n} p_{i}^{x}\right)^{1 / x}\left(\sum_{i=1}^{n} q_{i}^{y}\right)^{1 / y} \leq c_{x}(\phi, \varphi) \sum_{i=1}^{n} p_{i} q_{i}
$$

where $\quad c_{x}(\phi, \varphi)=\max \left\{1 / x(\phi / \varphi)^{1 / y}+1 / y(\varphi / \phi)^{1 / x}, 1 / x(\varphi /\right.$ $\left.\phi)^{1 / y}+1 / y(\phi / \varphi)^{1 / x}\right\}$ is a constant with some positive constants $\phi, \varphi$. If $p_{i}>0$ for some $1 \leq i \leq n$, then the equality holds if and only if $\phi=\varphi$ and $p_{i}^{x}=\phi q_{i}^{y}$ for every $1 \leq i \leq n$. Setting $p_{i}=\xi\left(u_{i} / \mathscr{G}\right), q_{i}=1$ and $x=4, y=4 / 3$ and also $\phi=\delta^{4}$, $\varphi=\Delta^{4}$, we have

$$
\left(\sum_{i=1}^{n} \xi^{4}\left(\frac{u_{i}}{\mathscr{G}}\right)\right)^{1 / 4}\left(\sum_{i=1}^{n} 1\right)^{4 / 3} \leq \max \left\{\frac{1}{4}\left(\frac{\delta^{4}}{\Delta^{4}}\right)^{4 / 3}+\frac{4}{3}\left(\frac{\Delta^{4}}{\delta^{4}}\right)^{1 / 4}, \frac{1}{4}\left(\frac{\Delta^{4}}{\delta^{4}}\right)^{4 / 3}+\frac{4}{3}\left(\frac{\delta^{4}}{\Delta^{4}}\right)^{1 / 4}\right\} \sum_{i=1}^{n} \xi \frac{u_{i}}{\mathscr{G}}
$$

so $(Y(\mathscr{G}))^{1 / 4} n^{4 / 3} \leq 2 m\left\{1 / 4(\Delta / \delta)^{16 / 3}+4 / 3 \delta / \Delta\right\}$, i.e., $Y(\mathscr{G}) \leq$ $16 m^{4} / \sqrt[3]{n^{16}}\left\{1 / 4(\Delta / \delta)^{16 / 3}+4 / 3 \delta / \Delta\right\}^{4}$. This completes the proof.

Theorem 9. For an $n$ vertex graph $\mathscr{G}$, we have $Y(\mathscr{G})$ $\leq(F(\mathscr{G}))^{4 / 3}$. The equality is satisfied when $\mathscr{G}$ is regular.

Proof. Let $x_{1}, x_{2}, \ldots, x_{n}$ be $n$ positive real numbers, and let $s, t$ be positive rational numbers. Then, by Jensen's inequality ([26]) $\left(\sum_{i=1}^{n} x_{i}^{t}\right)^{1 / t} \leq\left(\sum_{i=1}^{n} x_{i}^{s}\right)^{1 / s}$ if $t>s>0$. The equality holds iff $x_{1}=x_{2}=\cdots=x_{n}$. Considering $x_{i}=\xi\left(u_{i} / \mathscr{G}\right)$ for $t=4$, $s=3$, then we have $\left(\sum_{i=1}^{n} \xi^{4}\left(u_{i} / \mathscr{G}\right)\right)^{1 / 4} \leq\left(\sum_{i=1}^{n} \xi^{3}\left(u_{i} / \mathscr{G}\right)\right)^{1 / 3}$, that is, $Y(\mathscr{G}) \leq(F(\mathscr{G}))^{4 / 3}$.

Theorem 10. Let $\mathscr{G}$ be a $(n, m)$ graph. Then, $Y(\mathscr{G}) \leq\left(\delta^{2}+\right.$ $\left.\Delta^{2}\right) M_{1}(\mathscr{G})-n \delta^{2} \Delta^{2}$. The equality is attained when $\mathscr{G}$ is regular.

Proof. Suppose $x_{i}, y_{i}, h$ and $H$ are the positive real numbers such that $h x_{i} \leq y_{i} \leq H x_{i}$ for $i=1,2, \ldots, n$. Then, by DiazMetacalf inequality [27], $\quad \sum_{i=1}^{n} y_{i}^{2}+h H \sum_{i=1}^{n} \leq(h+H)$ $\sum_{i=1}^{n} x_{i} y_{i}$ ant the equality is attained if and only if $y_{i}=h x_{i}$ 
and $y_{i}=H a_{i}$. Now taking $x_{i}=1$ and $y_{i}=\xi^{2}\left(u_{i} / \mathscr{G}\right)$ and $h=\delta^{2}, H=\Delta$, we get

$$
\sum_{i=1}^{n} \xi^{4}\left(\frac{u_{i}}{\mathscr{G}}\right)+\delta^{2} \Delta^{2} \sum_{i=1}^{n} 1 \leq\left(\delta^{2}+\Delta^{2}\right) \sum_{i=1}^{n} \xi^{2}\left(\frac{u_{i}}{\mathscr{G}}\right) .
$$

Thus, $Y(\mathscr{G}) \leq\left(\delta^{2}+\Delta^{2}\right) M_{1}(\mathscr{G})-n \delta^{2} \Delta^{2}$.

Theorem 11. Let $\mathscr{G}$ be a graph whose number of vertices is $n$ and edges $m$. Then,

$$
Y(\mathscr{G}) \leq \frac{\beta(n)(\Delta-\delta)^{2}\left(\Delta^{2}+\Delta \delta+\delta^{2}\right)+2 m F(\mathscr{G})}{n} .
$$

The equality is attained iff $x_{1}=x_{2}=\cdots=x_{n}$ and $z_{1}=$ $z_{2}=\cdots=z_{n}$ and also $\beta(n)=n\lceil n / 2\rceil(1-1 / n\lceil n / 2\rceil)$, where $\lceil x\rceil$ is the largest integer greater than or equal to $x$.

Proof. Let $x_{i}$ and $z_{i}$ be positive real numbers for which there exist real constants $x, z, X$ and $Z$ such that $x \leq x_{i} \leq X$ and $z \leq z_{i} \leq Z$ for $1 \leq i \leq n$, respectively. Then, we have (discrete)
Gruss inequality ([27]) $\left|n \sum_{i=1}^{n} x_{i} z_{i}-\sum_{i=1}^{n} x_{i} \sum_{i=1}^{n} z_{i}\right| \leq \beta(n)$ $(X-x)(Y-y)$.

The equality controls iff $x_{1}=x_{2}=\cdots=x_{n}$ and $z_{1}=z_{2}=\cdots=z_{n}$.

By setting $x_{i}=\xi^{3}(u / \mathscr{G})$ and $z_{i}=\xi(u / \mathscr{G})$ for every $i=1,2, \ldots, n$, we have $X=\Delta^{3}$ and $x=\delta^{3}$.

Then, the inequality becomes

$$
n \sum_{i=1}^{n} \xi^{4}\left(\frac{u_{i}}{\mathscr{G}}\right)-\sum_{i=1}^{n} \xi^{3}\left(\frac{u_{i}}{\mathscr{G}}\right) \sum_{i=1}^{n} \xi\left(\frac{u_{i}}{\mathscr{G}}\right) \leq \beta(n)\left(\Delta^{3}-\delta^{3}\right)(\Delta-\delta) .
$$

So, $Y(\mathscr{G}) \leq \beta(n)(\Delta-\delta)^{2}\left(\Delta^{2}+\Delta \delta+\delta^{2}\right)+2 m F(\mathscr{G}) / n$. This completes our claim.

Corollary 2. Since $\beta(n) \leq n^{2} / 4$, therefore $Y(\mathscr{G}) \leq n^{2}(\Delta$ $-\delta)^{2}\left(\Delta^{2}+\Delta \delta+\delta^{2}\right)+8 m F(\mathscr{G}) / 4 n$.

Theorem 12. Let $\mathscr{G}$ be a $(n, m)$ graph. Then,

$$
\begin{aligned}
Y(\mathscr{G}) \leq & (3 \Delta+\delta) F(\mathscr{G})-\left\{(n-1)(\Delta-\delta)+3 \delta^{2}-3 \delta \Delta\right\} M_{1}(\mathscr{G})-(n-1)(2 m-n \Delta) \delta^{2}-4(n-2) m \delta \Delta \\
& +2 m \Delta^{3}+6 m \delta \Delta^{2}-\delta \Delta^{3} n
\end{aligned}
$$

with equality holds if and only if $\mathscr{G}$ is $(\Delta, \delta)$ biregular.

Proof. We have from [28] that $Y(\mathscr{G})=(n-1) F(\mathscr{G})-\bar{Y}(\mathscr{G})$, where $\bar{Y}(\mathscr{G})$ be the $Y$-coindex of $\mathscr{G}$. From [29], $F(\mathscr{G}) \leq M_{1}(\mathscr{G})(\Delta+2 \delta)-\delta^{2}(2 m-n \Delta)-4 m \delta \Delta$. Define by $X(\mathscr{G})=(n-1) \sum_{u \in V(\mathscr{G})}(\xi(u / \mathscr{G})-\Delta)^{2} \quad(\xi(u / \mathscr{G})-\delta)-$ $\sum_{u \in V(\mathscr{G})}(\xi(u / \mathscr{G})-\Delta)^{3}(\xi(u / \mathscr{G})-\delta)$. Since $X(\mathscr{G}) \geq 0$, we have $\bar{Y}(\mathscr{G}) \geq\left\{(n-1)(\delta+2 \Delta)+3 \Delta^{2}+3 \delta \Delta\right\} M_{1}(\mathscr{G})-(3 \Delta+$ $\delta) F(\mathscr{G})-2 m \Delta^{2}+\delta \Delta^{2}-4 m \delta \Delta-2 m \Delta^{3}-6 m \delta \Delta^{2}+\delta \Delta^{3} n$.

After simplification, we get the required result.

Theorem 13. Let $\mathscr{G}$ be a $(n, m)$ graph, we have

$$
Y(\mathscr{G}) \leq(2 x+\Delta+\delta) F(\mathscr{G})-\left(x^{2}+\delta \Delta\right) M_{1}(\mathscr{G})+2 m x\{2 \delta \Delta+x(\delta+\Delta)\}-x^{2} \delta \Delta .
$$

The equality holds when $\mathscr{G}$ be a $(\Delta, \delta)$ biregular graph and also $\delta \leq x \leq \Delta$, where $x$ be a positive real number.
Proof. Define by $F_{1}(\mathscr{G})=\sum_{u \in V(\mathscr{G})}[\xi(u / \mathscr{G})-x]^{2}[\xi(u / \mathscr{G})-$ $y][\xi(u / \mathscr{G})-z]$.Setting $\delta \leq x \leq \Delta, \quad y=\Delta$ and $z=\delta$, then $F_{1}(\mathscr{G}) \leq 0$. Thus,

$$
F_{1}(\mathscr{G})=\left[Y(\mathscr{G})-(2 x+\Delta+\delta) F(\mathscr{G})+\left(x^{2}+\delta \Delta\right) M_{1}(\mathscr{G})-2 m x\{2 \delta \Delta+x(\delta+\Delta)\}+x^{2} \delta \Delta\right] \leq 0
$$

Theorem 14. Let $\mathscr{G}$ be a $(n, m)$ graph. Then,

$$
\begin{aligned}
Y(\mathscr{G}) \leq & (4 \Delta-6) F(\mathscr{G})-\{\Delta(\Delta-1)+(2 \Delta-1)(2 \Delta-5)+(\Delta-2)(\Delta-3)\} M_{1}(\mathscr{G}) \\
& +2 m\{(\Delta-2)(\Delta-3)(2 \Delta-1)+\Delta(\Delta-1)(2 \Delta-5)\}-n w x y z .
\end{aligned}
$$


The equality occurs when $\mathscr{G}$ is a tetra-regular graph. Proof. Suppose that

$$
\begin{aligned}
F_{2}(\mathscr{G})= & \sum_{u \in V(\mathscr{G})}\left[\xi\left(\frac{u}{\mathscr{G}}\right)-w\right]\left[\xi\left(\frac{u}{\mathscr{G}}\right)-x\right]\left[\xi\left(\frac{u}{\mathscr{G}}\right)-y\right]\left[\xi\left(\frac{u}{\mathscr{G}}\right)-z\right] \\
= & Y(\mathscr{G})-(w+x+y+z) F(\mathscr{G})+\{x w+(w+x)(y+z)+y z\} M_{1}(\mathscr{G}) \\
& -2 m\{(w+x) y z+(y+z) x w\}+w x y z
\end{aligned}
$$

where $w, x, y, z$ are the positive real numbers.Setting $w=$ $\Delta, x=\Delta-1, y=\Delta-2$ and $z=\Delta-3$, then $F_{2}(G) \leq 0$. Therefore, we get the required result. The equality is satisfied when $\mathscr{G}$ is a tetra-regular graph.

Corollary 3. Let $\mathscr{G}$ be a graph with $n$ vertices and $m$ edges. Then, $Y(\mathscr{G}) \leq(3 \Delta+\delta) F(\mathscr{G})-3 \Delta(\Delta+\delta) M_{1}(\mathscr{G})+2 m \Delta^{2}(\Delta$
$+3 \delta)-\Delta^{3} \delta n$ and also $Y(\mathscr{G}) \leq(3 \delta+\Delta) F(\mathscr{G})-3 \delta(\delta+\Delta) M_{1}$ $(\mathscr{G})+2 m \delta^{2}(\delta+3 \Delta)-\delta^{3} \Delta n$ with equality holding when $\mathscr{G}$ is a $(\Delta, \delta)$ biregular graph.

Proof. Consider an auxiliary function $F_{3}(\mathscr{G})=\sum_{u \in V(\mathscr{G})}$ $[\xi(u / \mathscr{G})-x]^{3}[\xi(u / \mathscr{G})-y]$, where $x$ and $y$ are the real numbers. Thus,

$$
\begin{aligned}
F_{3}(\mathscr{G})= & \sum_{u \in V(\mathscr{G})}\left[\xi^{4}\left(\frac{u}{\mathscr{G}}\right)-(3 x+y) \xi^{3}\left(\frac{u}{\mathscr{G}}\right)+3 x(x+y) \xi^{2}\left(\frac{u}{\mathscr{G}}\right)-x^{2}(x+3 y) \xi\left(\frac{u}{\mathscr{G}}\right)+x^{3} y\right]=Y(\mathscr{G})-(3 x+y) \\
& \cdot F(\mathscr{G})+3 x(x+3 y) M_{1}(\mathscr{G})-2 m x^{2}(x+3 y)+x^{3} y n
\end{aligned}
$$

Taking $x=\Delta, y=\delta$ then $F_{3}(\mathscr{G}) \leq 0$ and $Y(\mathscr{G}) \leq(3 \Delta+$ $\delta) F(\mathscr{G})-3 \Delta(\Delta+\delta) M_{1}(\mathscr{G})+2 m \Delta^{2}(\Delta+3 \delta)-\Delta^{3} \delta n$. Also

Corollary 4. Let $\mathscr{G}$ be a graph of order $n$ and size $m$. Then, for $x=\delta$ and $y=\Delta$, we have $F_{3}(\mathscr{G}) \leq 0$. Thus, $Y(\mathscr{G}) \leq(3 \delta+$ $\Delta) F(\mathscr{G})-3 \delta(\delta+\Delta) M_{1}(\mathscr{G})+2 m \delta^{2}(\delta+3 \Delta)-\delta^{3} \Delta n$.

$$
\begin{aligned}
\bar{Y}(\mathscr{G}) \leq & \{(n-1)(3 \Delta-1)+3 \Delta(2 \Delta-1)\} M_{1}(\mathscr{G})-(4 \Delta-1) F(\mathscr{G})-2 m(n-1) \Delta(3 \Delta-2)-2 m \Delta^{2}(4 \Delta-3) \\
& +\Delta^{2}(\Delta-1)(n+\Delta-1) n
\end{aligned}
$$

where the equality is satisfied iff $\mathscr{G}$ is $a(\Delta, \Delta-1)$ biregular graph.
Proof. Define by $F_{4}(\mathscr{G})=(n-1) \sum_{u \in V(\mathscr{G})}[\xi(u / \mathscr{G})-\Delta]^{2}[\xi$ $(u / \mathscr{G})-(\Delta-1)]-\sum_{u \in V(\mathscr{G})}[\xi(u / \mathscr{G})-\Delta]^{3}[\xi(u / \mathscr{G})-(\Delta-1)]$

$$
\begin{aligned}
F_{4}(\mathscr{G})= & (n-1) \sum_{u \in V(\mathscr{G})}\left[\xi\left(\frac{u}{\mathscr{G}}\right)-\Delta\right]^{2}\left[\xi\left(\frac{u}{\mathscr{G}}\right)-(\Delta-1)\right]-\sum_{u \in V(\mathscr{G})}\left[\xi\left(\frac{u}{\mathscr{G}}\right)-\Delta\right]^{3}\left[\xi\left(\frac{u}{\mathscr{G}}\right)-(\Delta-1)\right] \\
\leq & (n-1)\left[F(\mathscr{G})-(3 \Delta-1) M_{1}(\mathscr{G})+2 m \Delta(3 \Delta-2)-\Delta^{2}(\Delta-1) n\right]-Y(\mathscr{G})+(4 \Delta-1) F(\mathscr{G})-3 \Delta(2 \Delta-1) \\
& \cdot M_{1}(\mathscr{G})+2 m \Delta^{2}(4 \Delta-3)-\Delta^{3}(\Delta-1) n .
\end{aligned}
$$

Since $F_{4}(\mathscr{G}) \leq 0$

$$
\begin{aligned}
\bar{Y}(\mathscr{G}) \leq & \{(n-1)(3 \Delta-1)+3 \Delta(2 \Delta-1)\} M_{1}(\mathscr{G})-(4 \Delta-1) F(\mathscr{G})-2 m(n-1) \Delta(3 \Delta-2)-2 m \Delta^{2}(4 \Delta-3) \\
& +\Delta^{2}(\Delta-1)(n+\Delta-1) n
\end{aligned}
$$


Corollary 5. If $\mathscr{G}$ is a graph with $n$ vertices and $m$ edges, the upper bounds of the $\bar{Y}(\mathscr{G})$ are given by $3 \Delta(n+2 \Delta-1)$ $M_{1}(\mathscr{G})-4 \Delta F(\mathscr{G})+n \Delta^{2}(n+\Delta-1)-2 m \Delta^{2}(3 n+4 \Delta-3)$. The equality holds if $\mathscr{G}$ is a regular graph.

Proof. Similarly, it is to be proved by defining $F_{5}(\mathscr{G})=$ $(n-1) \sum_{u \in V(\mathscr{G})}[\xi(u / \mathscr{G})-\Delta]^{3}-\sum_{u \in V(\mathscr{G})}[\xi(u / \mathscr{G})-\Delta]^{4}$. Obviously, $F_{5}(\mathscr{G}) \leq 0$.

In 2005, Klavzar et al. [30] introduced the generalized Sierpinski graph $g S(\mathscr{G}, t)$. It is obtained from $S(\mathscr{G}, t)$ by adding a new vertex $u$, called the special vertex of $g S(\mathscr{G}, t)$, and edges joining $u$ with all extreme vertices of $S(\mathscr{G}, t)$.
Theorem 15. Let $\mathscr{G}$ be a graph of order $n$ and size $m$ and let $g S(\mathscr{G}, t)$ be its generalized Sierpinski graph with dimension $t \geq 2$. Then, the YI of $g S(\mathscr{G}, t)$ is given by

$$
\begin{aligned}
Y(g S(\mathscr{G}, t)) \leq & 2 m \Delta^{3}\left(n^{t-1}+4 n^{t-2}+4 \beta(n)_{t-2}\right) \\
& +2 m\left(n^{t-2}+\beta(n)_{t-2}\right)\left(6 \Delta^{2}+4 \Delta+1\right) .
\end{aligned}
$$

The upper bound is achieved iff $\mathscr{G}$ is a $\Delta$-regular graph.

Proof. The YI of $g S(\mathscr{G}, t)$ can be defined as

$$
Y(g S(\mathscr{G}, t))=\sum_{u v \in E(\mathscr{G})} \sum_{i, j=0}^{1}\left|\xi\left(\frac{u}{\mathscr{G}}\right)+i, \xi\left(\frac{v}{\mathscr{G}}\right)+j\right|_{S(\mathscr{G}, t)}\left(\left(\xi\left(\frac{u}{\mathscr{G}}\right)+i\right)^{3}+\left(\xi\left(\frac{u}{\mathscr{G}}\right)+j\right)^{3}\right)
$$

By applying Lemma 7, we have

$$
\begin{aligned}
= & \sum_{u v \in E(\mathscr{G})}\left[n^{t-2}\left(n-\xi\left(\frac{u}{\mathscr{G}}\right)-\xi\left(\frac{v}{\mathscr{G}}\right)+\triangleright(u, v)\right)\left(\xi^{3}\left(\frac{u}{\mathscr{G}}\right)+\xi^{3}\left(\frac{v}{\mathscr{G}}\right)\right)\right. \\
& +\left(n^{t-2}\left(\xi\left(\frac{v}{\mathscr{G}}\right)-\triangleright(u, v)\right)-\beta(n)_{t-2} \xi\left(\frac{u}{\mathscr{G}}\right)\right)\left(\xi^{3}\left(\frac{u}{\mathscr{G}}\right)+\left(\xi\left(\frac{v}{\mathscr{G}}\right)+1\right)^{3}\right) \\
& +\left(n^{t-2}\left(\xi\left(\frac{u}{\mathscr{G}}\right)-\triangleright(u, v)\right)-\beta(n)_{t-2} \xi\left(\frac{v}{\mathscr{G}}\right)\right)\left(\left(\xi\left(\frac{u}{\mathscr{G}}\right)+1\right)^{3}+\xi^{3}\left(\frac{v}{\mathscr{G}}\right)\right) \\
& \left.+\left(n^{t-2}(\triangleright(u, v)+1)+\beta(n)_{t-2}\left(\xi\left(\frac{u}{\mathscr{G}}\right)+\xi\left(\frac{v}{\mathscr{G}}\right)+1\right)\right)\left(\left(\xi\left(\frac{u}{\mathscr{G}}\right)+1\right)^{3}+\left(\xi\left(\frac{v}{\mathscr{G}}\right)+1\right)^{3}\right)\right] \\
\leq & \sum_{u v \in E(G)}\left[n^{t-2}(n-2 \Delta+\triangleright(u, v))\left(2 \Delta^{3}\right)+2\left(n^{t-2}(\Delta-\triangleright(u, v))-\beta(n)_{t-2} \Delta\right)\left(\Delta^{3}+(\Delta+1)^{3}\right)\right. \\
& \left.+\left(n^{t-2}(\triangleright(u, v)+1)+\beta(n)_{t-2}(2 \Delta+1)\right)\left(2(\Delta+1)^{3}\right)\right] \\
= & 2 m \Delta^{3}\left(n^{t-1}+4 n^{t-2}+4 \beta(n)_{t-2}\right)+2 m\left(n^{t-2}+\beta(n)_{t-2}\right)\left(6 \Delta^{2}+4 \Delta+1\right) .
\end{aligned}
$$

\section{Some UB for YI under Some Graph Operations}

In this section, we derive some UB for YI under several graph operations. Let $\mathscr{G}_{i}$ be a graph with the vertex set $\left|V\left(\mathscr{G}_{i}\right)\right|=n_{i}$ and the edge set $\left|E\left(\mathscr{G}_{i}\right)\right|=m_{i}$ for $i=1,2$. For each $u \in V\left(\mathscr{G}_{1}\right)$ and $v \in V\left(\mathscr{G}_{2}\right)$, we get $\xi\left(u / \mathscr{G}_{1}\right) \leq \Delta\left(\mathscr{G}_{1}\right)$ and $\xi\left(v / \mathscr{G}_{2}\right) \leq \Delta\left(\mathscr{G}_{2}\right)$.

4.1. Cartesian Product. The Cartesian product ([31]) of $\mathscr{G}_{1}$ and $\mathscr{G}_{2}$, denoted by $\mathscr{G}_{1} \otimes \mathscr{G}_{2}$, is the graph with vertex set
$V\left(\mathscr{G}_{1} \otimes \mathscr{G}_{2}\right)=V\left(\mathscr{G}_{1}\right) \times V\left(\mathscr{G}_{2}\right)$ and its degree distribution is $\xi\left((u, v) / \mathscr{G}_{1} \otimes \mathscr{G}_{2}\right)=\xi\left(u / \mathscr{G}_{1}\right)+\xi\left(v / \mathscr{G}_{2}\right)$.

Theorem 16. The YI of $\mathscr{G}_{1} \otimes \mathscr{G}_{2}$ satisfies the following inequality:

$Y\left(\mathscr{G}_{1} \otimes \mathscr{G}_{2}\right) \leq n_{1} n_{2}\left[\Delta^{4}\left(\mathscr{G}_{1}\right)+\Delta^{4}\left(\mathscr{G}_{2}\right)+4 \Delta^{3}\left(\mathscr{G}_{1}\right) \Delta\left(\mathscr{G}_{2}\right)+\right.$ $\left.4 \Delta\left(\mathscr{G}_{1}\right) \Delta^{3}\left(\mathscr{G}_{2}\right)+6 \Delta^{2}\left(\mathscr{G}_{1}\right) \Delta^{2}\left(\mathscr{G}_{2}\right)\right]$ with equality occurring when $\mathscr{G}_{1}$ and $\mathscr{G}_{2}$ are regular graphs.

Proof. By the definition of $Y$-index, we have 


$$
\begin{aligned}
Y\left(\mathscr{G}_{1} \otimes \mathscr{G}_{2}\right) & =\sum_{(u, v) \in V\left(\mathscr{G}_{1} \times \mathscr{G}_{2}\right)}\left[\xi\left(\frac{(u, v)}{\mathscr{G}_{1} \times \mathscr{G}_{2}}\right)\right]^{4} \\
& =\sum_{u \in V\left(\mathscr{G}_{1}\right)} \sum_{v \in V\left(\mathscr{G}_{2}\right)}\left[\xi\left(\frac{u}{\mathscr{G}_{1}}\right)+\xi\left(\frac{v}{\mathscr{G}_{2}}\right)\right]^{4} \\
& \leq n_{1} n_{2}\left[\Delta^{4}\left(\mathscr{G}_{1}\right)+\Delta^{4}\left(\mathscr{G}_{2}\right)+4 \Delta^{3}\left(\mathscr{G}_{1}\right) \Delta\left(\mathscr{G}_{2}\right)+4 \Delta\left(\mathscr{G}_{1}\right) \Delta^{3}\left(\mathscr{G}_{2}\right)+6 \Delta^{2}\left(\mathscr{G}_{1}\right) \Delta^{2}\left(\mathscr{G}_{2}\right)\right] .
\end{aligned}
$$

The inequality must be equality if $\xi\left(u_{1} / \mathscr{G}_{1}\right)+\xi\left(v_{1} / \mathscr{G}_{2}\right)=$ $\xi\left(u_{2} / \mathscr{G}_{1}\right)+\xi\left(v_{2} / \mathscr{G}_{2}\right)$ for any $u_{1}, u_{2} \in V\left(\mathscr{G}_{1}\right)$ and $v_{1}, v_{2} \in V$ $\left(\mathscr{G}_{2}\right)$.

4.2. Join. The degree of a vertex $u$ for the join [32] of $\mathscr{G}_{1}$ and $\mathscr{G}_{2}$, denoted by $\mathscr{G}_{1}+\mathscr{G}_{2}$, is given by

$$
\xi\left(\frac{u}{\mathscr{G}_{1}}+\mathscr{G}_{2}\right)= \begin{cases}\xi\left(\frac{u}{\mathscr{G}_{1}}\right)+n_{2} & \text { if } u \in V\left(\mathscr{G}_{1}\right) \\ \xi\left(\frac{u}{\mathscr{G}_{2}}\right)+n_{1} & \text { if } u \in V\left(\mathscr{G}_{2}\right)\end{cases}
$$

Theorem 17. The UB on the $Y$-index of two graphs $\mathscr{G}_{1}$ and $\mathscr{G}_{2}$ for join is given by

$$
\begin{aligned}
Y\left(\mathscr{G}_{1}+\mathscr{G}_{2}\right) \leq & 2 m_{1}\left(\Delta\left(\mathscr{G}_{1}\right)+n_{2}\right)^{3}+2 m_{2}\left(\Delta\left(\mathscr{G}_{2}\right)+n_{1}\right)^{3} \\
& +n_{1} n_{2}\left[\left(\Delta\left(\mathscr{G}_{1}\right)+n_{2}\right)^{3}+\left(\Delta\left(\mathscr{G}_{2}\right)+n_{1}\right)^{3}\right] .
\end{aligned}
$$

The equality holds when $\mathscr{G}_{1}$ and $\mathscr{G}_{2}$ are regular graphs.

Proof. By the definition of the YI, we get

$$
\begin{aligned}
Y\left(\mathscr{G}_{1}+\mathscr{G}_{2}\right)= & \sum_{u v \in E\left(\mathscr{G}_{1}\right)}\left[\left(\xi\left(\frac{u}{\mathscr{G}_{1}}\right)+n_{2}\right)^{3}+\left(\xi\left(\frac{v}{\mathscr{G}_{1}}\right)+n_{2}\right)^{3}\right] \\
& +\sum_{u v \in E\left(\mathscr{G}_{2}\right)}\left[\left(\xi\left(\frac{u}{\mathscr{G}_{2}}\right)+n_{1}\right)^{3}+\left(\xi\left(\frac{v}{\mathscr{G}_{2}}\right)+n_{1}\right)^{3}\right] \\
& +\sum_{u \in V\left(\mathscr{G}_{1}\right)} \sum_{v \in V\left(\mathscr{G}_{2}\right)}\left[\left(\xi\left(\frac{u}{\mathscr{G}_{1}}\right)+n_{2}\right)^{3}+\left(\xi\left(\frac{v}{\mathscr{G}_{2}}\right)+n_{1}\right)^{3}\right] \\
\leq & 2 m_{1}\left(\Delta\left(\mathscr{G}_{1}\right)+n_{2}\right)^{3}+2 m_{2}\left(\Delta\left(\mathscr{G}_{2}\right)+n_{1}\right)^{3} \\
& +n_{1} n_{2}\left[\left(\Delta\left(\mathscr{G}_{1}\right)+n_{2}\right)^{3}+\left(\Delta\left(\mathscr{G}_{2}\right)+n_{1}\right)^{3}\right] .
\end{aligned}
$$

4.3. Composition. For the composition $\mathscr{G}_{1}\left[\mathscr{G}_{2}\right]$ of two graphs $\mathscr{G}_{1}$ and $\mathscr{G}_{2}$ [11], the degree of a vertex $(u, v) \in V$ $\left(\mathscr{G}_{1}\left[\mathscr{G}_{2}\right]\right)$ is given by $d\left((u, v) / \mathscr{G}_{1}\left[\mathscr{G}_{2}\right]\right)=n_{2} \xi\left(u / \mathscr{G}_{1}\right)+\xi$ $\left(v / \mathscr{G}_{2}\right)$.
Theorem 18. The UB of YI for $\mathscr{G}_{1}\left[\mathscr{G}_{2}\right]$ is given by $Y\left(\mathscr{G}_{1}\right.$ $\left.\left[\mathscr{G}_{2}\right]\right) \leq n_{2}^{5} \Delta^{4}\left(\mathscr{G}_{1}\right)+n_{1} n_{2} \Delta^{4} \quad\left(\mathscr{G}_{2}\right)+6 n_{1} n_{2}^{3} \Delta^{2}\left(\mathscr{G}_{1}\right) \Delta^{2}$ $\left(\mathscr{G}_{2}\right)+4 n_{1} n_{2}^{4} \Delta^{3}\left(\mathscr{G}_{1}\right) \Delta\left(\mathscr{G}_{2}\right)+4 n_{1} n_{2}^{2} \Delta\left(\mathscr{G}_{1}\right) \Delta^{3}$. The equality carries for $\mathscr{G}_{1}$ and $\mathscr{G}_{2}$ regular graphs.

Proof. From the definition of $Y$-index, we have

$$
\begin{aligned}
Y\left(\mathscr{G}_{1}\left[\mathscr{G}_{2}\right]\right) & =\sum_{u \in V\left(\mathscr{G}_{1}\right)} \sum_{v \in V\left(\mathscr{G}_{2}\right)}\left[n_{2} \xi\left(\frac{u}{\mathscr{G}_{1}}\right)+\xi\left(\frac{v}{\mathscr{G}_{2}}\right)\right]^{4} \\
& \leq n_{2}^{5} \Delta^{4}\left(\mathscr{G}_{1}\right)+n_{1} n_{2} \Delta^{4}\left(\mathscr{G}_{2}\right)+6 n_{1} n_{2}^{3} \Delta^{2}\left(\mathscr{G}_{1}\right) \Delta^{2}\left(\mathscr{G}_{2}\right)+4 n_{1} n_{2}^{4} \Delta^{3}\left(\mathscr{G}_{1}\right) \Delta\left(\mathscr{G}_{2}\right)+4 n_{1} n_{2}^{2} \Delta\left(\mathscr{G}_{1}\right) \Delta^{3} .
\end{aligned}
$$


4.4. Corona Product. For Corona product [33] of $\mathscr{G}_{1}$ and $\mathscr{G}_{2}$, denoted by $\mathscr{G}_{1} \diamond \mathscr{G}_{2}$, the degree of a vertex $u \in \mathscr{G}_{1} \diamond \mathscr{G}_{2}$ is given by

$$
\xi\left(\frac{u}{\mathscr{G}_{1} \diamond \mathscr{G}_{2}}\right)= \begin{cases}\xi\left(\frac{u}{\mathscr{G}_{1}}\right)+n_{2} & \text { if } u \in V\left(\mathscr{G}_{1}\right) \\ \xi\left(\frac{u}{\mathscr{G}_{2}}\right)+1 & \text { if } u \in V\left(\mathscr{G}_{2, i}\right), i=1,2, \ldots, n_{1}\end{cases}
$$

where $\mathscr{G}_{2, i}$ is the $i$-th copy of the graph $\mathscr{G}_{2}$.

Theorem 19. Let $\mathscr{G}=\mathscr{G}_{1} \diamond \mathscr{G}_{2}$ be the corona product of $\mathscr{G}_{1}$ and $\mathscr{G}_{2} . Y(\mathscr{G})$ satisfies the following inequalities $Y(\mathscr{G}) \leq n_{1}$
$\left[\Delta\left(\mathscr{G}_{1}\right)+n_{2}\right]^{4}+n_{1} n_{2}\left[\Delta\left(\mathscr{G}_{2}\right)+1\right]^{4}$. The equality holds when $\mathscr{G}_{1}$ and $\mathscr{G}_{2}$ are regular.

Proof. From definition of $Y I$, we have

$$
\begin{aligned}
Y\left(\mathscr{G}_{1} \diamond \mathscr{G}_{2}\right)= & \sum_{u \in V\left(\mathscr{G}_{1}\right)}\left(\xi\left(\frac{u}{\mathscr{G}_{1}}\right)+n_{2}\right)^{4}+n_{1} \sum_{u \in V\left(\mathscr{G}_{2}\right)}\left(\xi\left(\frac{u}{\mathscr{G}_{2}}\right)+1\right)^{4} \\
\leq & n_{1}\left[\Delta^{4}\left(\mathscr{G}_{1}\right)+4 n_{2} \Delta^{3}\left(\mathscr{G}_{1}\right)+6 n_{2}^{2} \Delta^{2}\left(\mathscr{G}_{1}\right)+4 n_{2}^{3} \Delta\left(\mathscr{G}_{1}\right)+n_{2}^{4}\right] \\
& +n_{1} n_{2}\left[\Delta^{4}\left(\mathscr{G}_{2}\right)+4 \Delta^{3}\left(\mathscr{G}_{2}\right)+6 \Delta\left(\mathscr{G}_{2}\right)+4 \Delta\left(\mathscr{G}_{2}\right)+1\right] .
\end{aligned}
$$

4.5. Strong Product. Consider $\xi\left((u, v) / \mathscr{G}_{1} * \mathscr{G}_{2}\right)=\xi(u /$ $\left.\mathscr{G}_{1}\right)+\xi\left(v / \mathscr{G}_{2}\right)+\xi\left(u / \mathscr{G}_{1}\right)\left(v / \mathscr{G}_{2}\right)$ as a degree distribution of a vertex $(u, v)$ in the strong product [11] $\mathscr{G}_{1} * \mathscr{G}_{2}$.
Theorem 20. The sharp UB of YI for $\mathscr{G}_{1} * \mathscr{G}_{2}$ is given by

$$
\begin{aligned}
Y\left(\mathscr{G}_{1} * \mathscr{G}_{2}\right) \leq & n_{1} n_{2}\left[\Delta^{4}\left(\mathscr{G}_{1}\right)+\Delta^{4}\left(\mathscr{G}_{2}\right)+\Delta^{4}\left(\mathscr{G}_{1}\right) \Delta^{4}\left(\mathscr{G}_{2}\right)\right. \\
& +6 \Delta^{2}\left(\mathscr{G}_{1}\right) \Delta^{2}\left(\mathscr{G}_{2}\right)\left(\Delta^{2}\left(\mathscr{G}_{1}\right)+\Delta^{2}\left(\mathscr{G}_{2}\right)+1\right) \\
& +4 \Delta\left(\mathscr{G}_{1}\right) \Delta\left(\mathscr{G}_{2}\right)\left(\Delta^{3}\left(\mathscr{G}_{1}\right)+\Delta^{3}\left(\mathscr{G}_{2}\right)+\Delta^{3}\left(\mathscr{G}_{1}\right) \Delta^{2}\left(\mathscr{G}_{2}\right)\right. \\
& \left.+\Delta^{2}\left(\mathscr{G}_{1}\right) \Delta^{3}\left(\mathscr{G}_{2}\right)+\Delta^{2}\left(\mathscr{G}_{1}\right)+\Delta^{2}\left(\mathscr{G}_{2}\right)\right) \\
& \left.+12 \Delta^{2}\left(\mathscr{G}_{1}\right) \Delta^{2}\left(\mathscr{G}_{2}\right)\left(\Delta\left(\mathscr{G}_{1}\right)+\Delta\left(\mathscr{G}_{2}\right)+\Delta\left(\mathscr{G}_{1}\right) \Delta\left(\mathscr{G}_{2}\right)\right)\right] .
\end{aligned}
$$

The equality occurs when $\mathscr{G}_{1}$ and $\mathscr{G}_{2}$ are regular.

\section{Application}

As an application, we compute the YI of $C_{80}$ Fullerene, by using Theorem 11. Fullerenes are the molecules such as cagelike polyhedra, containing solely carbon atoms. Fullerenes contain the networks of pentagons and hexagons. Here, we consider the fullerene $C_{80}$ such that molecules made up entirely of $n$ (natural number) carbon atoms contain twelve pentagonal sides and $(n / 2-10)$ hexagonal faces, where $n(\neq 22) \geq 20$. For the graph representing fullerene $C_{80}$ which is given in [34], we have $F\left(C_{80}\right)=2160$. The number of edges $(\mathrm{m})$ in fullerene $C_{80}$ is $m=n r / 2=80 \times 3 / 2=120$.
Thus, by Theorem 11, $Y(\mathscr{G}) \leq \beta(n)(\Delta-\delta)^{2}\left(\Delta^{2}+\Delta \delta+\delta^{2}\right)+$ $2 m F(\mathscr{G}) / n=2 \times 120 \times 2160 / 80=6480$.

\section{Conclusion}

The YI is one of the new chemical descriptors, which passes the test of having a highly correlation with the physiochemical properties it claims to describe in [11]. It comes as no surprise. Then, we determine some new UBs for the YI using various parameters such as order, size, maximum degree, minimum degree, distance $\kappa$-domination number, and some other topological indices. Furthermore, some sharp UB for the YI based on graph binary operations are obtained. At last, we consider an application for YI index of 
$C_{80}$ Fullerene. The appeal of computing the UB is of course their generality and simple proofs. Along in this line, determining new lower bounds for YI is considered to be studied in the future.

\section{Data Availability}

No data were used to support this study.

\section{Conflicts of Interest}

The authors declare that there are no conflicts of interest regarding the publication of this article.

\section{Acknowledgments}

The second author acknowledges the support of DST-FIST, New Delhi (India) (Sanction No. SR/FST/MS- I/2018/21) for carrying out this work.

\section{References}

[1] N. Trinajstic, Chemical Graph Theory, CRC Press, Boca Raton, FL, USA, 1993.

[2] L. Pei and X. Pan, "Extremal values on Zagreb indices of trees with given distance k-domination number," Journal of Inequalities and Applications, vol. 2018, p. 16, 2018.

[3] L. Pei and X. Pan, "The minimum eccentric distance sum of trees with given distance k-domination number," Discret. Math. Algorithms Appl.vol. 2020, p. 16, 2020.

[4] F. Harary, Graph Theory, Addison-Wesley Publishing Co., Boston, MA, USA, 1969.

[5] H. Wiener, "Structural determination of paraffin boiling points," Journal of the American Chemical Society, vol. 69, no. 1, pp. 17-20, 1947.

[6] I. Gutman and N. Trinajstić, "Graph theory and molecular orbitals. Total $\varphi$-electron energy of alternant hydrocarbons," Chemical Physics Letters, vol. 17, no. 4, pp. 535-538, 1972.

[7] X. Li and J. Zheng, "A unified approach to the extremal trees for different indices," MATCH Communications in Mathematical and in Computer Chemistry, vol. 54, pp. 195-208, 2005.

[8] B. Furtula and I. Gutman, "A forgotten topological index," Journal of Mathematical Chemistry, vol. 53, no. 4, pp. 11841190, 2015.

[9] J.-B. Liu, B. Ali, M. A. Malik, H. M. A. Siddiqui, and M. Imran, "Reformulated Zagreb indices of some derived graphs," Mathematics, vol. 7, no. 4, p. 366, 2019.

[10] A. Milicevic, S. Nikolic, and N. Trinajstic, "On reformulated Zagreb indices," Molecular Diversity, vol. 8, pp. 393-399, 2004.

[11] A. Alameri, N. Al-Naggar, M. Al-Rumaima, and M. Alsharafi, "Y-index of some graph operations," International Journal of Applied Engineering, vol. 15, pp. 173-179, 2020.

[12] D. Maji and G. Ghorai, "A novel graph invariant: the third leap Zagreb index under several graph operations," Discrete Mathematics, Algorithms and Applications, vol. 11, no. 5, pp. 1-16, 2019.

[13] D. Maji and G. Ghorai, "Computing F-index, coindex and Zagreb polynomials of the kth generalized transformation graphs," Heliyon, vol. 6, no. 12, Article ID e05781, 2020.

[14] D. Maji and G. Ghorai, "The first entire Zagreb index of various corona products and their bounds," Journal of Mathematical and Computational Science, vol. 11, no. 5, pp. 6018-6044, 2021.
[15] D. Maji, G. Ghorai, M. K. Mahmood, and M. A. Alam, "On the inverse problem for some topological indices," Journal of Mathematics, vol. 2021, no. 1, pp. 1-8, 2021.

[16] D. Maji, G. Ghorai, and Y. U. Gaba, "On the reformulated second Zagreb index of graph operations," Journal of Chemistry, vol. 2021, no. 5, pp. 1-17, 2021.

[17] F. Hayat, "On extremal results of multiplicative Zagreb indices of trees with given distance $k$-domination number," 2019, https://arxiv.org/abs/1906.12202.

[18] H. Hua, S. Zhang, and K. Xu, "Further results on the eccentric distance sum," Discrete Applied Mathematics, vol. 160, no. 1-2, pp. 170-180, 2012.

[19] A. Meir and J. Moon, "Relations between packing and covering numbers of a tree," Pacific Journal of Mathematics, vol. 61, no. 1, pp. 225-233, 1975.

[20] J. Topp and L. Volkmann, "On packing and covering numbers of graphs,” Discrete Mathematics, vol. 96, no. 3, pp. 229-238, 1991.

[21] M. Imran and M. K. Jamil, "Sharp bounds on certain degree based topological indices for generalized Sierpiński graphs," Chaos, Solitons \& Fractals, vol. 132, Article ID 109608, 2020.

[22] A. Ali, M. Metajic, M. Matejic, and I. Milovanovic, "Some new upper bounds for the inverse sum indeg index of graphs," Electronic Journal of Graph Theory and Applications, vol. 8, no. 1, pp. 59-70, 2020.

[23] B. Borovicanin and B. Furtula, "On extremal Zagreb indices of trees with given domination number," Applied Mathematics and Computation, vol. 279, pp. 208-218, 2016.

[24] B. Zhou and I. Gutman, "Further properties of Zagreb indices," MATCH Communications in Mathematical and in Computer Chemistry, vol. 54, pp. 233-239, 2005.

[25] P. Erdos and T. Gallai, "Graphs with prescribed degrees of vertices (in Hungarian)," Mat. Lapok.vol. 11, pp. 264-274, 1960.

[26] Z. Che and Z. Chem, "Lower and upper bounds of forgotten topological index," MATCH Communications in Mathematical and in Computer Chemistry, vol. 76, pp. 635-648, 2016.

[27] S. Hosamani and B. Basavanagoud, "New upper bounds for the first Zagreb index," MATCH Commun. Math. Comput. Chem.vol. 74, pp. 97-101, 2015.

[28] A. Alameri, M. Al-Rumaima, and M. Almazah, "Y-coindex of graph operations and its applications of molecular descriptors," Journal of Molecular Structure, vol. 1221, Article ID 128754, 2020.

[29] A. Ghalavand, A. Ashrafi, A. Ashrafi, and I. Gutman, "New upper and lower bounds for some degree-based graph invariants," Kragujevac Journal of Mathematics, vol. 44, no. 2, pp. 181-188, 2020.

[30] S. Klavar and B. Mohar, "Crossing numbers of Sierpinski-like graphs,” Journal of Graph Theory, vol. 50, pp. 186-198, 2005.

[31] S. Mondal, N. De, and A. Pal, "On neighborhood Zagreb index of product graphs," Journal of Molecular Structure, vol. 1223, Article ID 129210, 2021.

[32] M. Cancan, N. De, M. Alaeiyan, and M. Reza Farahan, "On reformulated narumi-katayama index," Proyecciones (Antofagasta), vol. 39, no. 5, pp. 1333-1346, 2020.

[33] K. Pattabiraman and P. Kandan, "Weighted PI index of corona product of graphs," Discrete Mathematics, Algorithms and Applications, vol. 6, no. 4, Article ID 1450055, 2014.

[34] S. Hosamani, A upper bound for the F1-index and its applications to fullerenes, 2015. 